\title{
Convergence Rates for Regularized Solutions
}

\author{
By Mark A. Lukas
}

Abstract. Given a first-kind integral equation

$$
\mathscr{K} u(x)=\int_{0}^{1} K(x, t) u(t) d t=f(x)
$$

with discrete noisy data $d_{i}=f\left(x_{i}\right)+\varepsilon_{i}, i=1,2, \ldots, n$, let $u_{n \alpha}$ be the minimizer in a Hilbert space $W$ of the regularization functional $(1 / n) \sum\left(\mathscr{K} u\left(x_{i}\right)-d_{i}\right)^{2}+\alpha\|u\|_{W}^{2}$. It is shown that in any one of a wide class of norms, which includes $\|\cdot\|_{W}$, if $\alpha \rightarrow 0$ in a certain way as $n \rightarrow \infty$, then $u_{n \alpha}$ converges to the true solution $u_{0}$. Convergence rates are obtained and are used to estimate the optimal regularization parameter $\alpha$.

1. Introduction. Consider the Fredholm integral equation of the first kind,

$$
\int_{0}^{1} K(x, t) u(t) d t=f(x), \quad x \in[0,1],
$$

where $f$ is given only as discrete noisy data

$$
d_{i}=f\left(x_{i}\right)+\varepsilon_{i}, \quad i=1,2, \ldots, n .
$$

The errors $\varepsilon_{i}$ are assumed to be uncorrelated random variables, each with mean 0 and variance $\sigma^{2}$.

For an approximate solution to this ill-posed problem, we use the following form of regularization:

$$
\underset{u \in W}{\operatorname{minimize}} \frac{1}{n} \sum_{i=1}^{n}\left(\mathscr{K} u\left(x_{i}\right)-d_{i}\right)^{2}+\alpha\|u\|_{W}^{2} .
$$

Here, $\alpha>0$ is called the regularization parameter and $\mathscr{K}$ is the integral operator defined by

$$
\mathscr{K} u(x)=\int_{0}^{1} K(x, t) u(t) d t
$$

The space $W$ is either $L^{2}[0,1]$ or a reproducing kernel Hilbert space (RKHS) of functions on $[0,1]$, i.e., a Hilbert space with the property that all the evaluation functionals $W \rightarrow \mathbf{R}, u \rightarrow u(x), x \in[0,1]$, are bounded. Their representer $R(x, t)$ is called the reproducing kernel (RK) of $W$ and satisfies $R_{x}=R(x, \cdot) \in W$ and $\left(R_{x}, u\right)_{W}=u(x)$ for all $x \in[0,1]$ and $u \in W$. It is not hard to show that the RK $R(x, t)$ is unique and symmetric. For examples and properties of these spaces, see [1] and [9].

Received May 29, 1984; revised September 12, 1986.

1980 Mathematics Subject Classification (1985 Revision). Primary 41A25, 45L05, 65R20. 
For the purpose of regularization, the best examples of RKHS are the Sobolev spaces of order $m=1,2, \ldots, W^{m, 2}[0,1]=\left\{u: u^{(m-1)}\right.$ is absolutely continuous and $\left.u^{(m)} \in L^{2}[0,1]\right\}$, with inner product and norm given by

$$
\begin{gathered}
(u, v)_{W^{m, 2}}=\gamma(u, v)+\left(u^{(m)}, v^{(m)}\right), \quad \gamma>0 \\
\|u\|_{W^{m, 2}}^{2}=\gamma\|u\|^{2}+\left\|u^{m}\right\|^{2}
\end{gathered}
$$

or perhaps with other equivalent norms. Here and throughout, $\|\cdot\|$ and $(\cdot, \cdot)$ denote the $L^{2}[0,1]$-norm and inner product. Also useful are closed subspaces of $W^{m, 2}$ such as

$$
W=\left\{u \in W^{2,2}[0,1]: u(0)=u(1)=0\right\}
$$

with

$$
(u, v)_{W}=\left(u^{\prime \prime}, v^{\prime \prime}\right), \quad\|u\|_{W}^{2}=\left\|u^{\prime \prime}\right\|^{2} .
$$

For the reproducing kerneis of these spaces, see [16] and [20].

To derive a solution to (1.1), we require that for each $x \in[0,1]$ the linear functional $W \rightarrow \mathbb{R}, u \rightarrow \mathscr{K} u(x)$ is bounded. With $W=L^{2}[0,1]$, this is true if for each $x,\|K(x, \cdot)\|<\infty$. If $W$ is a RKHS, it will be true if, for example, $R(t, t)$ is a bounded function (certainly the case if $W=W^{m, 2}[0,1]$ ) and for each $x, K(x, \cdot)$ is integrable. This follows since for all $u \in W$,

$$
|u(t)|=\left|\left(R_{t}, u\right)_{W}\right| \leq\left(R_{t}, R_{t}\right)_{W}^{1 / 2}\|u\|_{W}=R(t, t)^{1 / 2}\|u\|_{W}
$$

and then

$$
\begin{aligned}
|\mathscr{K} u(x)| & =\left|\int_{0}^{1} K(x, t) u(t) d t\right| \\
& \leq \sup _{t} R(t, t)^{1 / 2} \int_{0}^{1}|K(x, t)| d t\|u\|_{W} .
\end{aligned}
$$

We will assume that for each $x \in[0,1],\|K(x, \cdot)\|<\infty$, and that $K(x, t) \in L^{2}[0,1] \times$ $[0,1]$. This covers both cases above and also implies that $\mathscr{K}: L^{2}[0,1] \rightarrow L^{2}[0,1]$ is bounded.

Although integral equations of the first kind are the main application, the results of this paper are not restricted to these. In fact, they will apply to any operator equation $\mathscr{K} u=f$ such that the functionals $W \rightarrow \mathbf{R}, u \rightarrow \mathscr{K} u(x)$ are bounded.

Let $\eta_{x}$ be the representer of the functional $u \rightarrow \mathscr{K} u(x)$ so that for all $u \in W$, $\left(\eta_{x}, u\right)_{W}=\mathscr{K} u(x)$. If $W=L^{2}[0,1]$, then clearly $\eta_{x}(t)=K(x, t)$. If $W$ is a RKHS, then substituting $u=R_{t}$ gives

$$
\eta_{x}(t)=\left(\eta_{x}, R_{t}\right)=\mathscr{K} R_{t}(x)=\mathscr{R} K_{x}(t),
$$

where $\mathscr{R}$ is the integral operator with kernel $R(t, s)$.

Now the regularization problem (1.1) is known (see [21]) to have the unique solution, called the regularized solution,

$$
u_{n \alpha}=\eta\left(Q_{n}+\alpha n I\right)^{-1} \mathbf{d} .
$$

Here $\eta_{i}(t)=\eta_{x_{i}}(t)$ and $Q_{n}$ is the $n \times n$ matrix with entries

$$
\left[Q_{n}\right]_{i j}=\left(\eta_{i}, \eta_{j}\right)_{W}=\mathscr{K} \eta_{j}\left(x_{i}\right)=Q\left(x_{i}, x_{j}\right),
$$


where $Q(x, y)$ is the symmetric kernel

$$
\begin{aligned}
Q(x, y) & =\left(\eta_{x}, \eta_{y}\right)_{W} \\
& =\left\{\begin{array}{l}
K K^{*}(x, y)=\int_{0}^{1} K(x, t) K(y, t) d t, \quad W=L^{2}[0,1] \\
K R K^{*}(x, y)=\int_{0}^{1} \int_{0}^{1} K(x, t) R(t, s) K(y, s) d s d t,
\end{array} \quad W\right. \text { a RKHS. }
\end{aligned}
$$

From (1.2), note that

$$
\mathscr{K} u_{n \alpha}=\mathbf{Q}\left(Q_{n}+\alpha n I\right)^{-1} \mathbf{d}
$$

where

$$
Q_{i}(x)=Q\left(x_{i}, x\right)
$$

If $f \in \mathscr{K}(W)$, let $\mathscr{K}^{\dagger} f$ be the solution to $\mathscr{K} u=f$ of minimal $W$ norm. Equivalently, $\mathscr{K}^{\dagger} f$ is the unique solution to $\mathscr{K} u=f$ which is orthogonal in $W$ to the null space $N(\mathscr{K})$. Given any $u \in W$, there exist unique elements $u_{0} \in N(\mathscr{K})$ and $u_{1} \in N(\mathscr{K})^{\perp}$ such that $u=u_{0}+u_{1}$. Substituting $u=u_{0}+u_{1}$ into the regularization functional (1.1) shows that for a minimum we must have $u_{0}=0$. Thus, for all $n$ and $\alpha, u_{n \alpha} \in N(\mathscr{K})^{\perp}$. Since $N(\mathscr{K})^{\perp}$ is closed in $W$, if $u_{n \alpha}$ converges in $W$ to some solution of $\mathscr{K} u=f$, then that solution must be $\mathscr{K}^{\dagger} f$.

By considering (1.1), it is intuitively clear that if $\alpha$ is held fixed while $n \rightarrow$ $\infty$, then the term $\alpha\|u\|_{W}^{2}$ will prevent $u_{n \alpha}$ from converging to $\mathscr{K}^{\dagger} f$. To achieve convergence, the regularization parameter $\alpha=\alpha(n)$ must tend to 0 but, as we will see, it must not go to 0 too quickly. In this paper we show, under certain assumptions, that in any one of a wide class of norms, $u_{n \alpha}$ converges to $\mathscr{K}^{\dagger} f$ for a specific range of $\alpha=\alpha(n) \rightarrow 0$, and we also determine the convergence rates. We now proceed to define these norms.

Assume that the kernel $Q(x, y)$ is continuous on $[0,1] \times[0,1]$ and define $\mathscr{Q}: L^{2}[0,1]$ $\rightarrow L^{2}[0,1]$ by

$$
\mathscr{Q} f(x)=\int_{0}^{1} Q(x, y) f(y) d y
$$

Then clearly $\mathscr{Q}$ is bounded, selfadjoint and positive, i.e., for all $f \in L^{2}[0,1]$, $(\mathscr{Q} f, f) \geq 0$. To see the latter, note that for any $c_{i} \in \mathbb{R}$ and points $t_{i} \in[0,1]$, $i=1, \ldots, m$,

$$
\sum_{i, j=1}^{m} c_{i} Q\left(t_{i}, t_{j}\right) c_{j}=\left\|\sum_{i=1}^{m} c_{i} \eta_{t_{i}}\right\|_{W}^{2} \geq 0 .
$$

In particular, if $f \in C[0,1]$, then for any Riemann sum

$$
(\mathscr{Q} f, f)=\lim _{m \rightarrow \infty} \sum_{i, j=1}^{m} f\left(t_{i}\right) Q\left(t_{i}, t_{j}\right) f\left(t_{j}\right) \Delta t_{i} \Delta t_{j} \geq 0 .
$$

Since $C[0,1]$ is dense in $L^{2}[0,1]$ and $\mathscr{Q}$ is bounded, the inequality is true for all $f \in L^{2}[0,1]$.

Since $Q(x, y) \in L^{2}[0,1] \times[0,1], \mathscr{Q}$ is Hilbert-Schmidt and the theory for these operators (see [14]) yields the following. Other than a possible 0 eigenvalue and corresponding null space, $\mathscr{Q}$ has a (possibly finite) nonincreasing sequence of eigenvalues 
$\lambda_{i}>0$ (repeated according to their multiplicity) and corresponding orthonormal eigenfunctions $\phi_{i}$. These form a complete system in $N(\mathscr{Q})^{\perp} \subseteq L^{2}[0,1]$, and for any $f \in L^{2}[0,1]$

$$
\mathscr{Q} f=\sum_{i=1}^{\infty} \lambda_{i}\left(f, \phi_{i}\right) \phi_{i},
$$

where the sum converges in $L^{2}[0,1]$. Furthermore, since $Q(x, y)$ is continuous, $\phi_{i} \in$ $C[0,1]$ and, by Mercer's Theorem, $Q(x, y)$ has the uniformly convergent expansion

$$
Q(x, y)=\sum_{i=1}^{\infty} \lambda_{i} \phi_{i}(x) \phi_{i}(y)
$$

and

$$
\int_{0}^{1} Q(x, x) d x=\sum_{i=1}^{\infty} \lambda_{i} .
$$

Thus, $\mathscr{Q}$ is a trace class operator and it has a unique positive square root given by

$$
\mathscr{Q}^{1 / 2} f=\sum_{i=1}^{\infty} \lambda_{i}^{1 / 2}\left(f, \phi_{i}\right) \phi_{i} .
$$

Henceforth, we will assume that the nonzero eigenvalues $\lambda_{i}$ of $\mathscr{Q}$ decay like

$$
0<a_{1} i^{-2 p} \leq \lambda_{i} \leq a_{2} i^{-2 p}, \quad i=1,2, \ldots
$$

for some $p>1 / 2$.

Since $Q(x, y)$ satisfies (1.4), there exists (see [1]) a unique RKHS $H$ with RK $Q(x, y)$. Because $Q(x, y)$ is continuous on $[0,1] \times[0,1]$, it is not hard to show (see [20]) that $H \subset C[0,1]$. In fact, from [10], $H$ can be described as

$$
H=\mathscr{Q}^{1 / 2}\left(L^{2}[0,1]\right)=\left\{f \in N(\mathscr{Q})^{\perp} \subseteq L^{2}[0,1]: \sum_{i=1}^{\infty}\left(f, \phi_{i}\right)^{2} / \lambda_{i}<\infty\right\}
$$

with inner product

$$
(f, g)_{H}=\left(\mathscr{Q}^{1 / 2 \dagger} f, \mathscr{Q}^{1 / 2 \dagger} g\right)=\sum_{i=1}^{\infty}\left(f, \phi_{i}\right)\left(g, \phi_{i}\right) / \lambda_{i},
$$

and also as

$$
H=\mathscr{K}(W)
$$

with inner product

$$
(f, g)_{H}=\left(\mathscr{K}^{\dagger} f, \mathscr{K}^{\dagger} g\right)_{W} .
$$

The latter implies that $\mathscr{K}: N(\mathscr{K})^{\perp} \subseteq W \rightarrow H$ is an isometric isomorphism.

Now for $\mu \geq 0$, define

$$
H_{\mu}=\mathscr{Q}^{\mu / 2}\left(L^{2}[0,1]\right)=\left\{f \in N(\mathscr{Q})^{\perp} \subseteq L^{2}[0,1]: \sum_{i=1}^{\infty}\left(f, \phi_{i}\right)^{2} / \lambda_{i}^{\mu}<\infty\right\} .
$$

Then, with the inner product

$$
(f, g)_{H_{\mu}}=\sum_{i=1}^{\infty}\left(f, \phi_{i}\right)\left(g, \phi_{i}\right) / \lambda_{i}^{\mu},
$$


$H_{\mu}$ is a Hilbert space. The collection of $H_{\mu}, \mu \geq 0$, is called a scale of Hilbert spaces (see [17, p. 335]). For example, if the eigenfunctions $\phi_{i}$ are trigonometric and $N(\mathscr{Q})=0$, then

$$
H_{\mu}=\left\{f \in L^{2}[0,1]: \sum_{j=-\infty}^{\infty}\left|\hat{f}_{j}\right|^{2} j^{2 p \mu}<\infty\right\}
$$

where $\hat{f}_{j}$ is the $j$ th Fourier coefficient of $f$. But this is simply the periodic Sobolev space with fractional order of smoothness $p \mu$.

Define $W_{\mu}$ to be the Hilbert space completion of

$$
\left\{u \in N(\mathscr{K})^{\perp} \subseteq W: \mathscr{K} u \in H_{\mu}\right\}
$$

under the inner product

$$
(u, v)_{W_{\mu}}=(\mathscr{K} u, \mathscr{K} v)_{H_{\mu}}
$$

Note that $\mathscr{K}: W_{\mu} \rightarrow H_{\mu}$ is an isometric isomorphism. When it is clear from the function, we will write simply $\|f\|_{\mu}$ for $\|f\|_{H_{\mu}}$ if $f \in H_{\mu}$, and $\|u\|_{\mu}$ for $\|u\|_{W_{\mu}}$ if $u \in W_{\mu}$.

Note that $H_{1}=H$ and $W_{1}=N(\mathscr{K})^{\perp} \subseteq W$. In addition, $H_{0}=N(\mathscr{Q})^{\perp} \subseteq$ $L^{2}[0,1]$ and $W_{0}$ is even larger. In fact, $L^{2}[0,1]$ is continuously imbedded in $W_{0}$, since for all $u \in L^{2}[0,1]$,

$$
\|u\|_{W_{0}}=\|\mathscr{K} u\|_{H_{0}}=\|P \mathscr{K} u\| \leq\|\mathscr{K} u\| \leq\|\mathscr{K}\|\|u\| .
$$

Here, $P$ is the orthogonal projection of $L^{2}[0,1]$ onto $N(\mathscr{Q})^{\perp}$.

With $\mu$ as the index, the spaces $H_{\mu}$ and $W_{\mu}$ are ordered according to the strength of their norms - the larger is $\mu$ the stronger is the norm. For if $\nu<\mu$, then

$$
\|f\|_{H_{\nu}}^{2}=\sum_{i=1}^{\infty} \frac{\left(f, \phi_{i}\right)^{2}}{\lambda_{i}^{\nu}}=\sum_{i=1}^{\infty} \frac{\left(f, \phi_{i}\right)}{\lambda_{i}^{\mu}} \lambda_{i}^{\mu-\nu} \leq \max \left\{\lambda_{i}^{\mu-\nu}\right\}\|f\|_{H_{\mu}}^{2}
$$

and hence $H_{\mu} \subset H_{\nu}$ with continuous imbedding. This is a strict inclusion, since the function $f \in L^{2}[0,1]$ with $\left(f, \phi_{i}\right)^{2}=\lambda_{i}^{\mu} i^{-1}$ belongs in $H_{\nu}$ but not in $H_{\mu}$. Furthermore, if $\nu<\mu$ then $H_{\mu}$ is dense in $H_{\nu}$. This follows since, if $f \in H_{\nu}$ and $\varepsilon>0$, then there exists $f_{N}=\sum_{i=1}^{N}\left(f, \phi_{i}\right) \phi_{i} \in H_{\mu}$ such that $\left\|f-f_{N}\right\|_{\nu}<\varepsilon$.

Given $f \in H_{\mu}$, let $\mathscr{K}^{\dagger} f \in W_{\mu}$ denote the unique solution in $W_{\mu}$ to $\mathscr{K} u=f$. Note that when $\mu=1, \mathscr{K}^{\dagger} f$ is simply the solution to $\mathscr{K} u=f$ in $N(\mathscr{K})^{\perp} \subseteq W$. Now if $u_{n \alpha} \in W_{\mu}$, our problem is to estimate the expected squared error

$$
E\left\|u_{n \alpha}-\mathscr{K}^{\dagger} f\right\|_{W_{\mu}}^{2}=E\left\|\mathscr{K} u_{n \alpha}-f\right\|_{H_{\mu}}^{2} .
$$

This can be decomposed into the squared bias

$$
\left\|E u_{n \alpha}-\mathscr{K}^{\dagger} f\right\|_{W_{\mu}}^{2}=\left\|E \mathscr{K} u_{n \alpha}-f\right\|_{H_{\mu}}^{2}
$$

and the variance

$$
E\left\|u_{n \alpha}-E u_{n \alpha}\right\|_{W_{\mu}}^{2}=E\left\|\mathscr{K} u_{n \alpha}-E \mathscr{K} u_{n \alpha}\right\|_{H_{\mu}}^{2}
$$


as follows:

$$
\begin{aligned}
E\left\|\mathscr{K} u_{n \alpha}-f\right\|_{H_{\mu}}^{2}= & E\left\|E \mathscr{K} u_{n \alpha}-f+\mathscr{K} u_{n \alpha}-E \mathscr{K} u_{n \alpha}\right\|_{H_{\mu}}^{2} \\
= & E\left(E \mathscr{K} u_{n \alpha}-f, E \mathscr{K} u_{n \alpha}-f\right)_{H_{\mu}} \\
& +2 E\left(E \mathscr{K} u_{n \alpha}-f, \mathscr{K} u_{n \alpha}-E \mathscr{K} u_{n \alpha}\right)_{H_{\mu}} \\
& +E\left(\mathscr{K} u_{n \alpha}-E \mathscr{K} u_{n \alpha}, \mathscr{K} u_{n \alpha}-E \mathscr{K} u_{n \alpha}\right)_{H_{\mu}} \\
= & \left\|E \mathscr{K} u_{n \alpha}-f\right\|_{H_{\mu}}^{2}+E\left\|\mathscr{K} u_{n \alpha}-E \mathscr{K} u_{n \alpha}\right\|_{H_{\mu}}^{2},
\end{aligned}
$$

since from (1.3), for any $g \in H_{\mu}$,

$$
\begin{aligned}
E\left(g, \mathscr{K} u_{n \alpha}-E \mathscr{K} u_{n \alpha}\right)_{H_{\mu}} & =\sum_{i, j=1}^{n}\left(g, Q\left(x_{i}, \cdot\right)\right)_{H_{\mu}}\left(Q_{n}+\alpha n I\right)_{i j}^{-1} E\left(d_{j}-f\left(x_{j}\right)\right) \\
& =0 .
\end{aligned}
$$

Consider the following continuous regularization problem corresponding to (1.1):

$$
\underset{u \in W}{\operatorname{minimize}}\|\mathscr{K} u-f\|^{2}+\alpha\|u\|_{W}^{2} .
$$

It is known (see [8]) that this has the unique solution

$$
u_{\alpha}=\mathscr{R} \mathscr{K}^{*}(\mathscr{Q}+\alpha)^{-1} f
$$

and that

$$
\mathscr{K} u_{\alpha}=\mathscr{Q}(\mathscr{Q}+\alpha)^{-1} f=(\mathscr{Q}+\alpha)^{-1} \mathscr{Q} f .
$$

In Section 3 we derive estimates of the error

$$
\left\|u_{\alpha}-\mathscr{K}^{\dagger} f\right\|_{W_{\mu}}^{2}=\left\|\mathscr{K} u_{\alpha}-f\right\|_{H_{\mu}}^{2} .
$$

Although of interest on their own, these lead to estimates of the bias (1.6). For, in Section 4, we show that if $\alpha$ tends to 0 in a certain way, then we can bound the bias in terms of this error. In Section 5 we determine the asymptotic behavior of the variance (1.7). These results are combined, using (1.8), in Theorem 2.1, giving an estimate of the expected error (1.5) in regularization. From this, in Corollary 2.1 , we determine the optimal rate of convergence for $\alpha$.

It was shown by Wahba [21] that if $f \in H_{2}$ and $\alpha \rightarrow 0$ as $n \rightarrow \infty$ in such a way that $n \alpha^{1 / 2 p} \rightarrow \infty$, then

$$
E\left\|u_{n \alpha}-\mathscr{K}^{\dagger} f\right\|_{W}^{2} \leq\left(\alpha\|f\|_{H_{2}}^{2}+\frac{\sigma^{2}}{n} l_{p} \alpha^{-(2 p+1) / 2 p}\right)(1+o(1))
$$

where $l_{p}$ is a constant. In particular, if $\alpha^{*}=c n^{-2 p /(4 p+1)}$ for some constant $c$, then

$$
E\left\|u_{n \alpha^{*}}-\mathscr{K}^{\dagger} f\right\|_{W}^{2}=O\left(n^{-2 p /(4 p+1)}\right) .
$$

However, the proof required the strong assumption that in various expressions the eigenvalues $\lambda_{i}$ and eigenfunctions $\phi_{i}$ of $\mathscr{Q}$ can be used to approximate the eigenvalues $\lambda_{n i}^{2}$ and eigenvectors $\phi_{i}$ of the matrix $\frac{1}{n} Q_{n}$. In this paper, we derive the above result rigorously as a special case of Theorem 2.1 with $\mu=1$ and $s=2$.

Results similar to those derived here were recently and independently obtained by Cox [6]. However, his results are based on a different spectral decomposition (see p. 15 of [6]) to the one above and are not specifically applied to integral equations. Moreover, our method of proof is simpler and more direct. In addition, we find lower as well as upper estimates of the bias. 
For the special case of convolution integral equations with equally spaced data $d_{k}=f(k / n)+\varepsilon_{k}$, Rice and Rosenblatt [13] use Fourier series to derive convergence rates for a regularized solution. In the case of data smoothing, convergence results for the smoothing spline estimate have been obtained by Craven and Wahba [7], Utreras Diaz [18], [19], Speckman [15], Cox [4], [5] and Rice and Rosenblatt [12].

2. Main Results. In this section we state the major results of this paper and draw some conclusions about the optimal regularization parameter.

For easy reference, we list below our main assumptions.

Assumptions. 2.1. The errors $\varepsilon_{i}$ satisfy $E \varepsilon_{i}=0$ and $E \varepsilon_{i} \varepsilon_{j}=\sigma^{2} \delta_{i j}$.

2.2. The nonzero eigenvalues $\lambda_{i}$ of $\mathscr{Q}$ satisfy

$$
0<a_{1} i^{-2 p} \leq \lambda_{i} \leq a_{2} i^{-2 p} \text { for some constants } a_{1} \text { and } a_{2} \text {, and } p>\frac{1}{2} .
$$

2.3. There exists $\nu, 0<\nu<1-1 / 4 p$, and a sequence $k_{n} \rightarrow 0$ such that for all $f, g \in H$

$$
\left|\int_{0}^{1} f g-\frac{1}{n} \sum_{i=1}^{n} f\left(x_{i}\right) g\left(x_{i}\right)\right| \leq k_{n}\|f\|_{\nu}\|g\|_{\nu} .
$$

In Theorem 2.5 we find sufficient conditions for Assumption 2.3 to hold.

We will use the following notation. Given two positive functions $g(x)$ and $h(x)$, denote $g(x) \lesssim h(x)$ if there exists a constant $c$ such that $g(x) \leq \operatorname{ch}(x)$ for all $x$. Denote $g(x) \approx h(x)$ if $h(x) \lesssim g(x) \lesssim h(x)$. Note that $g(x) \approx h(x)$ is equivalent to $h(x) \approx g(x)$. We will also use the asymptotic relation $g(x) \sim h(x)$ which means, if say $x \rightarrow 0$, that $g(x)=h(x)(1+o(1))$ where $o(1) \rightarrow 0$ as $x \rightarrow 0$.

THEOREM 2.1. With Assumptions 2.1, 2.2 and 2.3, let $f \in H_{s}$, where $s \geq$ $\max \{\nu, \mu\}$ and $\mu<2-\nu-1 / 2 p$. Suppose that $\alpha=\alpha(n) \rightarrow 0$ as $n \rightarrow \infty$ in such $a$ way that

$$
k_{n} \alpha^{-\nu-1 / 4 p} \rightarrow 0
$$

and, if $\mu>\nu, s>\nu+2$,

$$
k_{n} \alpha^{-\nu / 2-\mu / 2-1 / 4 p} \rightarrow 0
$$

Then, for $\mu \leq s \leq \mu+2$,

$$
\alpha^{2}\|f\|_{\mu}^{2}+\frac{\sigma^{2}}{n} \alpha^{-\mu-1 / 2 p} \lesssim E\left\|u_{n \alpha}-\mathscr{K}^{\dagger} f\right\|_{\mu}^{2} \lesssim \alpha^{s-\mu}\|f\|_{s}^{2}+\frac{\sigma^{2}}{n} \alpha^{-\mu-1 / 2 p},
$$

and for $s \geq \mu+2$,

$$
E\left\|u_{n \alpha}-\mathscr{K}^{\dagger} f\right\|_{\mu}^{2} \approx \alpha^{2}\|f\|_{\mu+2}^{2}+\frac{\sigma^{2}}{n} \alpha^{-\mu-1 / 2 p} .
$$

Proof. Combine the estimates in Theorems 3.1, 4.1 and 5.1, using (1.8).

This result is similar to Theorem 3.1 of Cox [6]. However, the latter theorem, when applied to integral equations of the first kind, does not immediately give the above result because it employs a different spectral decomposition. Furthermore, our proof of Theorem 2.1 is simpler and more direct.

For a given $f$, define

$$
\bar{s}=\sup \left\{s: f \in H_{s}\right\}
$$


if the sup exists. Later we show by example that the lower bound in (2.1) cannot be strengthened to read: for any $\varepsilon>0$

$$
\alpha^{\bar{s}-\mu+\varepsilon}+\frac{\sigma^{2}}{n} \alpha^{-\mu-1 / 2 p} \lesssim E\left\|u_{n \alpha}-\mathscr{K}^{\dagger} f\right\|_{\mu}^{2} .
$$

Nevertheless, we can prove the following

THEOREM 2.2. Suppose that Assumptions 2.1, 2.2 and 2.3 hold and let $f \in H_{s}$, where $s \geq \max \{\nu, \mu\}, \bar{s} \leq \mu+2$, and $\mu<2-\nu-1 / 2 p$. For any $\varepsilon>0$, there exists a sequence $\alpha=\alpha(n) \rightarrow 0$ as $n \rightarrow \infty$ such that

$$
\alpha^{\bar{s}-\mu+\varepsilon}+\frac{\sigma^{2}}{n} \alpha^{-\mu-1 / 2 p} \lesssim E\left\|u_{n \alpha}-\mathscr{K}^{\dagger} f\right\|_{\mu}^{2} .
$$

Proof. Combine the estimates in Theorems 4.2 and 5.1, using (1.8).

With an extra assumption on $f$, the lower bound of Theorem 2.2 is valid for all sufficiently small $\alpha$.

THEOREM 2.3. Suppose that Assumptions 2.1, 2.2 and 2.3 hold and let $f \in H_{s}$, where $s \geq \max \{\nu, \mu\}, \bar{s} \leq \mu+2$, and $\mu<2-\nu-1 / 2 p$. Assume that, given $\varepsilon>0$, there exist $L$ and $C>0$ such that for all $N$

$$
\sum_{i=N}^{L N} \frac{\left(f, \phi_{i}\right)^{2}}{\lambda_{i}^{\bar{s}+\varepsilon}} \geq C N^{-2 p \varepsilon} .
$$

Suppose that $\alpha=\alpha(n) \rightarrow 0$ as $n \rightarrow \infty$ in such a way that

$$
k_{n} \alpha^{-\nu-1 / 4 p-\varepsilon} \rightarrow 0 \text { when } \mu \leq \nu \text { or when } \mu>\nu, \bar{s} \leq \nu+2 \text {, }
$$

and

$$
k_{n} \alpha^{-\nu / 2-\mu / 2-1 / 4 p-\varepsilon / 2} \rightarrow 0 \quad \text { when } \mu>\nu, \bar{s}>\nu+2
$$

Then,

$$
\alpha^{\bar{s}-\mu+\varepsilon}+\frac{\sigma^{2}}{n} \alpha^{-\mu-1 / 2 p} \lesssim E\left\|u_{n \alpha}-\mathscr{K}^{\dagger} f\right\|_{\mu}^{2} .
$$

Proof. Combine the estimates in Theorems 3.3, 4.3 and 5.1, using (1.8).

The condition (2.2) on $f$ is quite general. It is satisfied, for example, if

$$
\frac{\left(f, \phi_{i}\right)^{2}}{\lambda_{i}^{\bar{s}}} \gtrsim i^{-1-4 p \varepsilon} \text {. }
$$

It can easily be shown that the set of all $f \in H_{s}$ satisfying (2.2) is dense in $H_{s}$.

If $f$ satisfies $\left(f, \phi_{i}\right)^{2} \approx \lambda_{i}^{r}$, then $\bar{s}=r-1 / 2 p$ and $f$ also satisfies condition (2.2). In fact, for this special case the following estimates can be obtained.

THEOREM 2.4. Suppose that Assumptions 2.1, 2.2 and 2.3 hold, and assume that $f$ satisfies $\left(f, \phi_{i}\right)^{2} \approx \lambda_{i}^{r}$. If, for some $\varepsilon>0, \alpha=\alpha(n) \rightarrow 0$ as in Theorem 2.3, then

where $\bar{s}=r-1 / 2 p$.

$$
E\left\|u_{n \alpha}-\mathscr{K}^{\dagger} f\right\|_{\mu}^{2} \approx \begin{cases}\alpha^{\bar{s}-\mu}+\frac{\sigma^{2}}{n} \alpha^{-\mu-1 / 2 p}, & \mu<\bar{s}<\mu+2, \\ \alpha^{2} \ln \frac{1}{\alpha}+\frac{\sigma^{2}}{n} \alpha^{-\mu-1 / 2 p}, & \bar{s}=\mu+2, \\ \alpha^{2}+\frac{\sigma^{2}}{n} \alpha^{-\mu-1 / 2 p}, & \bar{s}>\mu+2,\end{cases}
$$


Proof. Combine the estimates in Theorems 3.4, 4.3 and 5.1, using (1.8).

From Theorem 2.1 we have the following

COROLLARY 2.1. The minimum of the upper bounds in Theorem 2.1 occurs at

$$
\alpha^{*} \approx \begin{cases}\left(\frac{\sigma^{2}}{n}\right)^{2 p /(2 p s+1)}, & \mu<s \leq \mu+2, \\ \left(\frac{\sigma^{2}}{n}\right)^{2 p /(4 p+2 p \mu+1)}, & s \geq \mu+2 .\end{cases}
$$

With $\alpha=\alpha^{*}$, the upper and lower bounds in Theorem 2.1 have the same order, and if $\alpha^{*} \rightarrow 0$ as in Theorem 2.1, then

$$
E\left\|u_{n \alpha^{*}}-\mathscr{K}^{\dagger} f\right\|_{\mu}^{2} \approx \begin{cases}\left(\frac{\sigma^{2}}{n}\right)^{2 p(s-\mu) /(2 p s+1)}, & \mu<s \leq \mu+2, \\ \left(\frac{\sigma^{2}}{n}\right)^{4 p /(4 p+2 p \mu+1)}, & s \geq \mu+2 .\end{cases}
$$

It is clear that to make full use of Corollary 2.1, one should take $s$ as large as is allowed by $f \in H_{s}$. This gives both a weaker condition on $k_{n}$ and, if $s \leq \mu+2$, a better convergence rate for the error.

Let

$$
e_{n \alpha}=E\left\|u_{n \alpha}-\mathscr{K}^{\dagger} f\right\|_{\mu}^{2}
$$

and suppose that $\bar{\alpha}=\bar{\alpha}(n)$ minimizes $e_{n \alpha}$. We say that $\alpha=\alpha(n)$ is optimal if $e_{n \alpha} \approx e_{n \bar{\alpha}}$.

COROLlaRY 2.2. Suppose $f \in H_{s}$, where $s>\max \{\nu, \mu\}$ and $\mu<2-\nu-1 / 2 p$.

(a) Assume that as $n \rightarrow \infty, \alpha=\alpha(n) \rightarrow 0$ as in Theorem 2.1.

(i) If $s \geq \mu+2$, then $\alpha$ is optimal if and only if

$$
\alpha \approx\left(\frac{\sigma^{2}}{n}\right)^{2 p /(4 p+2 p \mu+1)}
$$

and then

$$
E\left\|u_{n \alpha}-\mathscr{K}^{\dagger} f\right\|_{\mu}^{2} \approx\left(\frac{\sigma^{2}}{n}\right)^{4 p /(4 p+2 p \mu+1)} .
$$

(ii) Suppose $s<\mu+2$ and $\bar{s} \leq \mu+2$. If $\alpha$ is optimal, then for any $0<\varepsilon<\bar{s}-\mu$,

$$
\alpha \gtrsim\left(\frac{\sigma^{2}}{n}\right)^{2 p /(2 p(\bar{s}-\varepsilon)+1)} \text {. }
$$

(b) Suppose $s<\mu+2$ and $\bar{s} \leq \mu+2$, and let $0<\varepsilon<\bar{s}-\mu$. Assume that $f$ satisfies condition (2.2) and that as $n \rightarrow \infty, \alpha=\alpha(n) \rightarrow 0$ as in Theorem 2.3. If $\alpha$ is optimal, then

$$
\alpha \lesssim\left(\frac{\sigma^{2}}{n}\right)^{2 p /(2 p(\bar{s}+\varepsilon)+1)} .
$$

Thus, from the class $\left\{\alpha: \alpha \approx\left(\sigma^{2} / n\right)^{t}, t \in \mathbf{R}\right\}$, the optimal $\alpha$ is

$$
\alpha \approx\left(\frac{\sigma^{2}}{n}\right)^{2 p /(2 p \bar{s}+1)} \text {. }
$$

Furthermore, for any sufficiently small $\delta>0$,

$$
\left(\frac{\sigma^{2}}{n}\right)^{2 p(\bar{s}-\mu) /(2 p \bar{s}+1)+\delta} \lesssim E\left\|u_{n \alpha}-\mathscr{K}^{\dagger} f\right\|_{\mu}^{2} \lesssim\left(\frac{\sigma^{2}}{n}\right)^{2 p(\bar{s}-\mu) /(2 p \bar{s}+1)-\delta} .
$$


(c) Assume that $f$ satisfies $\left(f, \phi_{i}\right)^{2} \approx \lambda_{i}^{r}$ and $\bar{s}=r-1 / 2 p>\mu$. If, for some $\varepsilon>0, \alpha=\alpha(n) \rightarrow 0$ as in Theorem 2.3, then

$$
\alpha \approx \begin{cases}\left(\frac{\sigma^{2}}{n}\right)^{2 p /(2 p \bar{s}+1)}, & \mu<\bar{s}<\mu+2, \\ \left(\frac{\sigma^{2}}{n}\right)^{2 p /(4 p+2 p \mu+1)}, & \bar{s}>\mu+2\end{cases}
$$

is optimal and

$$
E\left\|u_{n \alpha}-\mathscr{K}^{\dagger} f\right\|_{\mu}^{2} \approx \begin{cases}\left(\frac{\sigma^{2}}{n}\right)^{2 p(\bar{s}-\mu) /(2 p \bar{s}+1)}, & \mu<\bar{s}<\mu+2, \\ \left(\frac{\sigma^{2}}{n}\right)^{4 p /(4 p+2 p \mu+1)}, & \bar{s}>\mu+2 .\end{cases}
$$

If $\bar{s}=\mu+2$ and $\alpha$ solves

$$
\alpha^{(4 p+2 p \mu+1) / 2 p} \ln \frac{1}{\alpha} \approx \frac{\sigma^{2}}{n}
$$

then $\alpha$ is optimal.

Proof. (a)(i) From Theorem 2.1 and Corollary 2.1, it is clear that $e_{n \bar{\alpha}} \approx e_{n \alpha^{*}}$, so $\alpha^{*}$ is optimal. Now suppose $\alpha$ is optimal and let $\alpha=C_{n} \alpha^{*}$. Then

$$
\left(C_{n}^{2}+C_{n}^{-\mu-1 / 2 p}\right)\left(\alpha^{*}\right)^{2} \approx e_{n \alpha} \approx e_{n \alpha^{*}} \approx\left(\alpha^{*}\right)^{2} .
$$

This implies $C_{n} \approx 1$, so $\alpha \approx \alpha^{*}$.

(a)(ii) Let $s=\bar{s}-\varepsilon$ and suppose that $\alpha$ is optimal. If (2.5) were not true, then there would exist a subsequence $n_{i}$ such that, as $i \rightarrow \infty$,

$$
\theta_{i} \equiv \alpha\left(n_{i}\right)\left(\frac{\sigma^{2}}{n_{i}}\right)^{-2 p /(2 p s+1)} \rightarrow 0 .
$$

Substituting $\alpha=\alpha\left(n_{i}\right)$ into (2.1) gives

$$
e_{n_{i} \alpha\left(n_{i}\right)} \approx \theta_{i}^{-\mu-1 / 2 p} e_{n_{i} \alpha^{*}\left(n_{i}\right)}
$$

This shows that $\alpha$ is not optimal, a contradiction.

(b) This follows by a similar argument to that in (a)(ii).

(c) This follows directly from Theorem 2.4 .

Note that from Corollary 2.2(c), if $f$ satisfies $\left(f, \phi_{i}\right)^{2} \approx \lambda_{i}^{r}$ with $\bar{s}=r-1 / 2 p<2$, then the rate of convergence of the optimal $\alpha$ is independent of $\mu$. Therefore, if one has a good estimate of the optimal $\alpha$ for the $L^{2}$-norm $(\mu=0)$, then the regularized solution should be accurate in all $W_{\mu}$-norms, $0 \leq \mu<\bar{s}$. This behavior has been observed in practice by Wahba [22]. On the other hand, from Corollary 2.2(a)(i), for arbitrary $f$ with $\bar{s}>2$ the rate of convergence of the optimal $\alpha$ is dependent on $\mu$ for $\mu$ satisfying $0 \leq \mu \leq \bar{s}-2$.

Lastly, in this section we find sufficient conditions for Assumption 2.3 to hold. One possible approach to this question is to make a smoothness assumption on the $\nu$ th root of $Q(x, y)$. However, this approach appears to be impractical.

A practical approach to the verification of Assumption 2.3 is developed by Cox in Section 4 of [6]. In our notation it proceeds as follows. 
Suppose that we can find $l \geq 1$ such that $\mathscr{K}: W \rightarrow W^{l, 2}$ is bounded. Then for $f=\mathscr{K} u, g=\mathscr{K} v \in H=\mathscr{K}(W)$ we have $f, g \in W^{1,2}$, and it is not difficult to verify that

$$
\left|\int_{0}^{1} f g-\frac{1}{n} \sum_{i=1}^{n} f\left(x_{i}\right) g\left(x_{i}\right)\right| \leq d_{n}\|f\|_{W^{1,2}}\|g\|_{W^{1,2}}
$$

where

$$
d_{n}=\sup _{[0,1]}\left|x-F_{n}(x)\right|, \quad F_{n}(x)=\sum_{i: x_{i} \leq x} \frac{1}{n} .
$$

Now, from interpolation theory (see [17]) it is known that the intermediate spaces between $L^{2}$ and $W^{l, 2}$ are

$$
\left(L^{2}, W^{l, 2}\right)_{\theta, 2}=W^{\theta l, 2}, \quad 0 \leq \theta \leq 1 .
$$

Here, $W^{\theta l, 2}$ is the Sobolev space of fractional order $\theta l$ (which equals the Besov space $B_{2,2}^{\theta l}$ ). It is also known (see [2, p. 244]) that the intermediate spaces between $W_{0}$ and $W$ are

$$
\left(W_{0}, W\right)_{\theta, 2}=W_{\theta}, \quad 0 \leq \theta \leq 1 .
$$

Therefore, because both $\mathscr{K}: W \rightarrow W^{l, 2}$ and $\mathscr{K}: W_{0} \rightarrow L^{2}$ are bounded, then for any $k, \mathscr{K}: W_{k / l} \rightarrow W^{k, 2}$ is bounded, too. Hence, with $k=1$, there is a constant $c$ such that

$$
\|f\|_{W^{1,2}}=\|\mathscr{K} u\|_{W^{1,2}} \leq c\|u\|_{1 / l}=c\|f\|_{1 / l}
$$

and similarly for $g$.

Substituting these bounds into (2.6) yields Assumption 2.3 with $k_{n}=c^{2} d_{n}$ and $\nu=1 / l$. However, because the assumption requires $\nu<1-(1 / 4 p)$, then for integral $l$ we actually need $l \geq 2$.

It is not hard to see that if the points $x_{i}$ are uniformly spaced as $x_{i}=(i-a) / n$, $0 \leq a \leq 1$, then $d_{n}=O\left(n^{-1}\right)$, and this is the best possible order for $d_{n}$. In this case, with a further assumption on $\mathscr{K}$, we show in Theorem 2.5 that it is possible to improve on Cox's estimate.

First, define the Sobolev space of periodic functions by

$$
W_{\mathrm{per}}^{m, 2}=\left\{u \in W^{m, 2}[0,1]: u^{(i)}(0)=u^{(i)}(1), i=0, \ldots, m-1\right\}
$$

with inner product

$$
(u, v)_{\mathrm{per}}=\int_{0}^{1} u v+\int_{0}^{1} u^{(m)} v^{(m)}
$$

or, equivalently, by

$$
W_{\text {per }}^{m, 2}=\left\{u \in L^{2}[0,1]: \sum_{j=-\infty}^{\infty}\left|\hat{u}_{j}\right|^{2}(2 \pi j)^{2 m}<\infty\right\}
$$

with inner product

$$
(u, v)_{\mathrm{per}}=\int_{0}^{1} u v+\sum_{j=-\infty}^{\infty} \hat{u}_{j} \overline{\hat{v}}_{j}(2 \pi j)^{2 m} .
$$

Here, $\hat{u}_{j}$ denotes the $j$ th Fourier coefficient of $u$. For nonintegral $m$, the second definition defines the periodic Sobolev space of fractional order $m$. 
THEOREM 2.5. If $x_{i}=(i-a) / n, i=1, \ldots, n, 0 \leq a \leq 1$, and $\mathscr{K}: W \rightarrow W_{\text {per }}^{\beta, 2}$, $\beta>1 / 2$, is bounded, then Assumption 2.3 holds with $\nu=\gamma / \beta$ and $k_{n} \lesssim n^{-\gamma}$ for any $1 / 2<\gamma \leq \beta$.

Proof. Denote

$$
D(f)=\int_{0}^{1} f-\frac{1}{n} \sum_{i=1}^{n} f\left(x_{i}\right) .
$$

If $f \in H$, then $f \in W_{\mathrm{per}}^{\beta, 2}$, and by expanding $f$ in a Fourier series it is easy to show that

$$
D(f)=-\sum_{j \neq 0} \hat{f}_{j n} e^{-2 \pi i a j} .
$$

Then, for any $1 / 2<\gamma \leq \beta$,

$$
\begin{aligned}
|D(f)| & =\left|\sum_{j \neq 0} \hat{f}_{j n} e^{-2 \pi i a j}(j n)^{\gamma}(j n)^{-\gamma}\right| \\
& \leq\left[\sum_{j \neq 0}\left|\hat{f}_{j n}\right|^{2}(j n)^{2 \gamma}\right]^{1 / 2}\left[\sum_{j \neq 0}(j n)^{-2 \gamma}\right]^{1 / 2} \\
& \leq\|f\|_{W_{\text {per }}^{\gamma, 2} c_{1} n^{-\gamma}}
\end{aligned}
$$

where

$$
c_{1}=c_{1}(\gamma)=\left[\sum_{j \neq 0} j^{-2 \gamma}\right]^{1 / 2} .
$$

Because $W_{\text {per }}^{\gamma, 2}, \gamma>1 / 2$, is a Banach algebra (see [3]), we also have for $f, g \in H \subseteq$ $W_{\text {per }}^{\gamma, 2}$

$$
|D(f g)| \leq c_{2} n^{-\gamma}\|f\|_{W_{\mathrm{per}}^{\gamma, 2}}\|g\|_{W_{\mathrm{per}}^{\gamma, 2}} .
$$

Now, since $\mathscr{K}: W \rightarrow W_{\text {per }}^{\beta, 2}$ and $\mathscr{K}: W_{0} \rightarrow L^{2}$ are bounded, interpolation theory implies that $\mathscr{K}: W_{\gamma / \beta} \rightarrow W_{\text {per }}^{\gamma, 2}$ is bounded. Therefore, for some constant $c_{3}$,

$$
\|f\|_{W_{\mathrm{per}}^{\gamma, 2}}^{\gamma} \leq c_{3}\|f\|_{\gamma / \beta}
$$

and similarly for $g$. Substituting these bounds into the bound on $|D(f g)|$ proves the result.

3. Estimates of the Continuous Regularization Error. In this section we derive upper and lower estimates of the error $\left\|u_{\alpha}-\mathscr{K}^{\dagger} f\right\|_{\mu}$, where $u_{\alpha}$ solves the continuous regularization problem (1.9).

We will see that estimates in this and later sections depend on the behavior of the sum

$$
S(\alpha)=\sum_{i=1}^{\infty} \frac{\lambda_{i}^{2-z}}{\left(\lambda_{i}+\alpha\right)^{2}}
$$

With Assumption 2.2 on the eigenvalues $\lambda_{i}$, this behavior can be determined as follows. For the proof, see Lemma 2.1 of [6]. 
LEMMA 3.1. If $z<2-1 / 2 p$ then $S(\alpha)$ is finite for any $\alpha>0$. In this case, as $\alpha \rightarrow 0$,

$$
S(\alpha) \approx T(\alpha)= \begin{cases}\alpha^{-z-1 / 2 p}, & \text { if }-1 / 2 p<z<2-1 / 2 p \\ \ln \frac{1}{\alpha}, & \text { if } z=-1 / 2 p \\ 1, & \text { if } z<-1 / 2 p .\end{cases}
$$

In fact, as $\alpha \rightarrow 0$,

$$
c_{1}(z) \leq S(\alpha)(T(\alpha))^{-1} \leq c_{2}(z)
$$

where

$$
\begin{aligned}
& c_{1}(z)=\left(a_{1} / a_{2}\right)^{2-z} a_{2}^{1 / 2 p} I(z)(1+o(1)), \\
& c_{2}(z)=\left(a_{2} / a_{1}\right)^{2-z} a_{1}^{1 / 2 p} I(z)(1+o(1))
\end{aligned}
$$

and

$$
I(z)= \begin{cases}\int_{0}^{\infty} \frac{x^{2 p z}}{\left(1+x^{2 p}\right)^{2}} d x \quad & \text { if }-1 / 2 p<z<2-1 / 2 p \\ 1 & \text { if } z=-1 / 2 p .\end{cases}
$$

If $z<-1 / 2 p$, then $S(\alpha) \uparrow \sum_{i=1}^{\infty} \lambda_{i}^{-z} \equiv c(z)$.

Suppose that $f \in H_{s}$ for some $s \geq 0$. It is possible, of course, that $f$ belongs to $H_{s}$ for all $s \geq 0$. This is the case, for example, if $f$ is a finite linear combination of $\phi_{i}, i=1,2, \ldots$, or if $\left(f, \phi_{i}\right)^{2}$ decreases exponentially. If this is not the case, then $\bar{s}<\infty$. Note that $f$ may or may not belong to $H_{\bar{s}}$. If, for example,

$$
\frac{\left(f, \phi_{i}\right)^{2}}{\lambda_{i}^{\bar{s}}}=\frac{1}{i(\ln i)^{r}}, \quad i \geq 2
$$

then $f \in H_{\bar{s}}$ for $r>1$ and $f \notin H_{\bar{s}}$ for $r \leq 1$.

THEOREM 3.1. Under Assumption 2.2, if $f \in H_{s}$, then

$$
\alpha^{2}\|f\|_{\mu}^{2} \lesssim\left\|u_{\alpha}-\mathscr{K}^{\dagger} f\right\|_{\mu}^{2} \lesssim \begin{cases}o(1), & s=\mu \\ \alpha^{s-\mu}\|f\|_{s}^{2}, & \mu<s \leq \mu+2, \\ \alpha^{2}\|f\|_{\mu+2}^{2}, & s \geq \mu+2 .\end{cases}
$$

In fact, if $s \geq \mu+2$, then

Proof. We have

$$
\left\|u_{\alpha}-\mathscr{K}^{\dagger} f\right\|_{\mu}^{2} \sim \alpha^{2}\|f\|_{\mu+2}^{2} .
$$

$$
\begin{aligned}
\left\|\mathscr{K} u_{\alpha}-f\right\|_{\mu}^{2} & =\left\|\mathscr{Q}(\mathscr{Q}+\alpha)^{-1} f-f\right\|_{\mu}^{2}=\alpha^{2}\left\|(\mathscr{Q}+\alpha)^{-1} f\right\|_{\mu}^{2} \\
& =\alpha^{2} \sum_{i=1}^{\infty} \frac{\left(f, \phi_{i}\right)^{2}}{\left(\lambda_{i}+\alpha\right)^{2}} \cdot \frac{1}{\lambda_{i}^{\mu}}=\alpha^{2} \sum_{i=1}^{\infty} \frac{\left(f, \phi_{i}\right)^{2}}{\lambda_{i}^{s}} \cdot \frac{\lambda_{i}^{s-\mu}}{\left(\lambda_{i}+\alpha\right)^{2}} \\
& \leq \alpha^{s-\mu}\left(\frac{a_{2}}{a_{1}}\right)^{s-\mu} \sum_{i=1}^{\infty} \frac{\left(f, \phi_{i}\right)^{2}}{\lambda_{i}^{s}} \cdot \frac{(\beta i)^{4 p+2 p \mu-2 p s}}{\left(1+(\beta i)^{2 p}\right)^{2}}
\end{aligned}
$$

where $\beta=\left(\alpha / a_{1}\right)^{1 / 2 p}$.

Clearly, from (3.1), $\left\|\mathscr{K} u_{\alpha}-f\right\|_{\mu}^{2} \gtrsim \alpha^{2}\|f\|_{\mu}^{2}$.

If $s=\mu$, then clearly

$$
\left\|\mathscr{K} u_{\alpha}-f\right\|_{\mu}^{2}=\sum_{i=1}^{\infty} \frac{\left(f, \phi_{i}\right)^{2}}{\lambda_{i}^{s}}\left(\frac{\alpha}{\lambda_{i}+\alpha}\right)^{2}
$$

decreases to 0 as $\alpha$ decreases to 0 . 
If $\mu \leq s \leq \mu+2$, then it is not hard to verify that the function

$$
g(t)=\frac{(\beta t)^{4 p+2 p \mu-2 p s}}{\left(1+(\beta t)^{2 p}\right)^{2}}, \quad t \geq 0,
$$

has maximum value $\leq 1$. Thus,

$$
\left\|\mathscr{K} u_{\alpha}-f\right\|_{\mu}^{2} \leq\left(a_{2} / a_{1}\right)^{s-\mu} \alpha^{s-\mu}\|f\|_{s}^{2} .
$$

If $s \geq \mu+2$, then from (3.1)

$$
\left\|\mathscr{K} u_{\alpha}-f\right\|_{\mu}^{2} \leq \alpha^{2}\|f\|_{\mu+2}^{2}
$$

and furthermore

$$
\left\|\mathscr{K} u_{\alpha}-f\right\|_{\mu}^{2} \sim \alpha^{2}\|f\|_{\mu+2}^{2} .
$$

A result similar to Theorem 3.1, under different assumptions, can be found in [11]. Next we have the following lower bounds.

THEOREM 3.2. Under Assumption 2.2,

$$
\left\|u_{\alpha}-\mathscr{K}^{\dagger} f\right\|_{\mu}^{2} \gtrsim \begin{cases}\alpha^{\bar{s}-\mu} F(\alpha), & \mu<\bar{s}<\mu+2 \quad\left(\text { also } \bar{s}=\mu \text { if } f \in H_{\bar{s}}\right), \\ \alpha^{2} B(\alpha), & \bar{s}=\mu+2, f \notin H_{\bar{s}}, \\ \alpha^{2}\|f\|_{\mu+2}^{2}, & \bar{s}>\mu+2 \quad\left(\text { also } \bar{s}=\mu+2 \text { if } f \in H_{\bar{s}}\right),\end{cases}
$$

where $F(\alpha)$ satisfies: For any $c>0$ and $\varepsilon>0, F(\alpha) \geq c \alpha^{\varepsilon}$ for an infinite sequence of $\alpha \rightarrow 0$. Also here, $B(\alpha) \uparrow \infty$ as $\alpha \rightarrow 0$ and satisfies $B(\alpha) \leq \alpha^{-\varepsilon}\|f\|_{\mu+2-\varepsilon}^{2}$.

Proof. Let $\beta=\left(\alpha / a_{2}\right)^{1 / 2 p}$ and $N=[1 / \beta]+1$. Then, for any $\varepsilon>0$,

$$
\begin{aligned}
\left\|\mathscr{K} u_{\alpha}-f\right\|_{\mu}^{2} & =\alpha^{2} \sum_{i=1}^{\infty} \frac{\left(f, \phi_{i}\right)^{2}}{\lambda_{i}^{\mu}} \frac{1}{\left(\lambda_{i}+\alpha\right)^{2}} \geq \frac{1}{4} \sum_{i=N}^{\infty} \frac{\left(f, \phi_{i}\right)^{2}}{\lambda_{i}^{\mu}} \\
& \geq \frac{1}{4}\left(\frac{a_{1}}{9^{p} a_{2}}\right)^{\bar{s}-\mu+\varepsilon / 2} \alpha^{\bar{s}-\mu+\varepsilon / 2} \sum_{i=N}^{2 N-1} \frac{\left(f, \phi_{i}\right)^{2}}{\lambda_{i}^{\bar{s}+\varepsilon / 2}}, \quad \beta \leq 1 .
\end{aligned}
$$

Now we claim that for any $C$ and $\delta>0$,

$$
G(N) \equiv \sum_{i=N}^{2 N-1} \frac{\left(f, \phi_{i}\right)^{2}}{\lambda_{i}^{\bar{s}+\varepsilon / 2}} \geq C N^{-\delta}
$$

for infinitely many $N$. If not, then there exists $C>0$ and $\delta>0$ such that for all but finitely many $N, G(N)<C N^{-\delta}$. Hence, there exists $D$ such that for all $N$, $G(N)<D N^{-\delta}$. Then

$$
\sum_{i=1}^{2^{K}} \frac{\left(f, \phi_{i}\right)^{2}}{\lambda_{i}^{\bar{s}+\varepsilon / 2}}<\sum_{k=0}^{K} G\left(2^{k}\right)<D \sum_{k=0}^{K} 2^{-\delta k} .
$$

Since the series on the right converges, while the series on the left diverges, we have the desired contradiction.

Putting $\delta=p \varepsilon$ and $F(\alpha)=\alpha^{\varepsilon / 2} G(N)$ gives the first bound. Note that if $f \notin H_{\bar{s}}$, then we need not have included the exponent $\varepsilon / 2$ in (3.2). Instead, simply put $\delta=2 p \varepsilon$.

In the case $\bar{s}=\mu+2$ and $f \notin H_{\bar{s}}$,

$$
\left\|\mathscr{K} u_{\alpha}-f\right\|_{\mu}^{2}=\alpha^{2} B(\alpha),
$$


where clearly

$$
B(\alpha)=\sum_{i=1}^{\infty} \frac{\left(f, \phi_{i}\right)^{2}}{\lambda_{i}^{\mu}} \frac{1}{\left(\lambda_{i}+\alpha\right)^{2}} \uparrow \infty \quad \text { as } \alpha \downarrow 0
$$

and

$$
B(\alpha) \leq \alpha^{-\varepsilon}\|f\|_{\mu+2-\varepsilon}^{2} .
$$

The third bound is obvious from Theorem 3.1.

Theorem 3.2 cannot be strengthened to read: If $\mu<\bar{s}<\mu+2$, then for any $\varepsilon>0$

$$
\left\|\mathscr{K} u_{\alpha}-f\right\|_{\mu} \gtrsim \alpha^{\bar{s}-\mu+\varepsilon}
$$

for all sufficiently small $\alpha$. To see this, let $\beta=\left(\alpha / a_{1}\right)^{1 / 2 p}$ and

$$
g_{\beta}(t)=\frac{(\beta t)^{4 p+2 p \mu-2 p \bar{s}}}{\left(1+(\beta t)^{2 p}\right)^{2}}, \quad \mu<\bar{s}<\mu+2 .
$$

Then, as in the proof of Theorem 3.1,

$$
\left\|\mathscr{K} u_{\alpha}-f\right\|_{\mu}^{2} \leq\left(a_{2} / a_{1}\right)^{\bar{s}-\mu} \alpha^{\bar{s}-\mu} \sum_{i=1}^{\infty} \frac{\left(f, \phi_{i}\right)^{2}}{\lambda_{i}^{\bar{s}}} g_{\beta}(i) .
$$

Now define a function $f$ as follows: Let $k_{l}$ be an increasing sequence of positive integers such that $J=\left\{j=0,1,2, \ldots: j \notin\left[k_{l}-1, k_{l}+1\right], l=1,2, \ldots\right\}$ is infinite, and suppose

$$
\frac{\left(f, \phi_{i}\right)^{2}}{\lambda_{i}^{\bar{s}}}= \begin{cases}1, & i=2^{2^{j}}, j \in J \\ 0, & \text { otherwise. }\end{cases}
$$

For $l=1,2, \ldots$, let $k=k_{l}$ and $\beta=2^{-2^{k}}$. Then, with $a=4 p+2 p \mu-2 p \bar{s}>0$ and $b=2 p \bar{s}-2 p \mu>0$, it is not difficult to show that

$$
\begin{aligned}
\sum_{i=1}^{\infty} \frac{\left(f, \phi_{i}\right)^{2}}{\lambda_{i}^{\bar{s}}} g_{\beta}(i) & \leq \sum_{j=0}^{k-2} g_{\beta}\left(2^{2^{j}}\right)+\sum_{j=k+2}^{\infty} g_{\beta}\left(2^{2^{j}}\right) \\
& \leq \frac{1}{a(\ln 2)^{2}} \beta^{a / 2}+\frac{1}{b(\ln 2)^{2}} \beta^{b}=O\left(\alpha^{c}\right)
\end{aligned}
$$

where $c=\min \{a / 4 p, b / 2 p\}$. Thus, for this example, (3.3) does not hold.

With an extra assumption on $f,(3.3)$ does hold.

THEOREM 3.3. Under Assumption 2.2, if $\mu<\bar{s} \leq \mu+2$ and $f$ satisfies condition (2.2), then for all sufficiently small $\alpha$,

$$
\left\|u_{\alpha}-\mathscr{K}^{\dagger} f\right\|_{\mu}^{2} \gtrsim \alpha^{\bar{s}-\mu+\varepsilon} .
$$

Proof. If $N=\left[\left(\alpha / a_{2}\right)^{-1 / 2 p}\right]+1$, then as in the proof of Theorem 3.2,

$$
\begin{aligned}
\left\|\mathscr{K} u_{\alpha}-f\right\|_{\mu}^{2} & =\alpha^{2} \sum_{i=1}^{\infty} \frac{\left(f, \phi_{i}\right)^{2}}{\lambda_{i}^{\mu}} \frac{1}{\left(\lambda_{i}+\alpha\right)^{2}} \\
& \gtrsim \alpha^{\bar{s}-\mu+\varepsilon / 2} \sum_{i=N}^{L N} \frac{\left(f, \phi_{i}\right)^{2}}{\lambda_{i}^{\bar{s}+\varepsilon / 2}}
\end{aligned}
$$

and the result follows. 
In Theorems 3.1, 3.2 and 3.3, the bounds cannot in general be improved to have the exponent of $\alpha$ equal to $\bar{s}-\mu$. To see this for the lower bound, in the case $f \in H_{\bar{s}}$, consider a function $f$ such that

$$
\frac{\left(f, \phi_{i}\right)^{2}}{\lambda_{i}^{\bar{s}}}=\frac{1}{i \ln i}, \quad i \geq 2 .
$$

It can be shown that for this example

$$
\left\|\mathscr{K} u_{\alpha}-f\right\|_{\mu}^{2} \approx \begin{cases}\alpha^{\bar{s}-\mu}\left(\ln \frac{1}{\alpha}\right)^{-1}, & \mu<\bar{s}<\mu+2 \\ \alpha^{2} \ln \left(\ln \frac{1}{\alpha}\right), & \bar{s}=\mu+2\end{cases}
$$

In the case $f \in H_{\bar{s}}$, a function $f$ such that

$$
\frac{\left(f, \phi_{i}\right)^{2}}{\lambda_{i}^{\bar{s}}}=\frac{1}{i(\ln i)^{2}}, \quad i \geq 2
$$

gives

$$
\left\|\mathscr{K} u_{\alpha}-f\right\|_{\mu}^{2} \approx \begin{cases}\alpha^{\bar{s}-\mu}\left(\ln \frac{1}{\alpha}\right)^{-2}, & \mu<\bar{s}<\mu+2, \\ \left(\ln \frac{1}{\alpha}\right)^{-1}, & \bar{s}=\mu .\end{cases}
$$

For the upper bound, consider a function $f$ with

$$
\frac{\left(f, \phi_{i}\right)^{2}}{\lambda_{i}^{\bar{s}}}=\frac{\ln i}{i}
$$

Then,

$$
\left\|\mathscr{K} u_{\alpha}-f\right\|_{\mu}^{2} \approx \begin{cases}\alpha^{\bar{s}-\mu} \ln \frac{1}{\alpha}, & \mu<\bar{s}<\mu+2, \\ \alpha^{2}\left(\ln \frac{1}{\alpha}\right)^{2}, & \bar{s}=\mu+2 .\end{cases}
$$

Exact error estimates can be obtained in the following special case.

THEOREM 3.4. Under Assumption 2.2, if $f$ satisfies $\left(f, \phi_{i}\right)^{2} \approx \lambda_{i}^{r}, r>\mu+$ $1 / 2 p$, then $\bar{s}=r-1 / 2 p$ and

$$
\left\|u_{\alpha}-\mathscr{K}^{\dagger} f\right\|_{\mu}^{2} \approx \begin{cases}\alpha^{\bar{s}-\mu}, & \mu<\bar{s}<\mu+2 \\ \alpha^{2} \ln (1 / \alpha), & \bar{s}=\mu+2 \\ \alpha^{2}, & \bar{s}>\mu+2\end{cases}
$$

Proof. The result follows immediately from (3.1) and Lemma 3.1.

4. Estimates of the Bias. We begin this section by expressing $\mathscr{K} u_{n \alpha}$ in a form suitable for estimation. Define an operator $\mathscr{Q}_{n}$ by

$$
\mathscr{Q}_{n} f(x)=\frac{1}{n} \sum_{i=1}^{n} Q\left(x, x_{i}\right) f\left(x_{i}\right)
$$

LEMMA 4.1. For all $n$ and $\alpha>0$,

$$
\mathscr{K} u_{n \alpha}=\mathscr{Q}_{n}\left(\mathscr{Q}_{n}+\alpha\right)^{-1} d=\left(\mathscr{Q}_{n}+\alpha\right)^{-1} \mathscr{Q}_{n} d,
$$

where $d(x)$ is any interpolant of d. Also,

$$
E \mathscr{K} u_{n \alpha}=\mathscr{Q}_{n}\left(\mathscr{Q}_{n}+\alpha\right)^{-1} f=\left(\mathscr{Q}_{n}+\alpha\right)^{-1} \mathscr{Q}_{n} f
$$


Proof. For any $g \in H$,

$$
\begin{aligned}
\left(\mathscr{Q}_{n} g, g\right)_{H} & =\frac{1}{n} \sum_{i=1}^{n} g\left(x_{i}\right)\left(Q\left(\cdot, x_{i}\right), g(\cdot)\right)_{H} \\
& =\frac{1}{n} \sum_{i=1}^{n} g^{2}\left(x_{i}\right) \geq 0 .
\end{aligned}
$$

Therefore, $\mathscr{Q}_{n}: H \rightarrow H$ is a positive operator. If $\left(\mathscr{Q}_{n}+\alpha\right) f=0$ for any function $f$, then

$$
\left(\mathscr{Q}_{n}\left(\mathscr{Q}_{n} f\right), \mathscr{Q}_{n} f\right)_{H}+\alpha\left(\mathscr{Q}_{n} f, \mathscr{Q}_{n} f\right)_{H}=0 .
$$

This implies, from above with $g=\mathscr{Q}_{n} f$, that $\mathscr{Q}_{n} f=0$. Hence, $\alpha f=0$ and so $f=0$. Thus, $\mathscr{Q}_{n}+\alpha$ has an inverse.

To see that $\mathscr{K} u_{n \alpha}=\left(\mathscr{Q}_{n}+\alpha\right)^{-1} \mathscr{Q}_{n} d$, note that from (1.3),

$$
\begin{aligned}
\left(\mathscr{Q}_{n}+\alpha\right) \mathscr{K} u_{n \alpha}(x)= & \left(\mathscr{Q}_{n}+\alpha\right) \sum_{j=1}^{n} Q\left(x, x_{j}\right)\left(Q_{n}+\alpha n I\right)^{-1} \mathbf{d}_{j} \\
= & \frac{1}{n} \sum_{i, j=1}^{n} Q\left(x, x_{i}\right) Q\left(x_{i}, x_{j}\right)\left(Q_{n}+\alpha n I\right)^{-1} \mathbf{d}_{j} \\
& +\alpha \sum_{i=1}^{n} Q\left(x, x_{i}\right)\left(Q_{n}+\alpha n I\right)^{-1} \mathbf{d}_{i} \\
= & \frac{1}{n} \sum_{i=1}^{n} Q\left(x, x_{i}\right)\left(Q_{n}+\alpha n I\right)\left(Q_{n}+\alpha n I\right)^{-1} \mathbf{d}_{i} \\
= & \mathscr{Q}_{n} d .
\end{aligned}
$$

Then also $\mathscr{K} u_{n \alpha}=\mathscr{Q}_{n}\left(\mathscr{Q}_{n}+\alpha\right)^{-1} d$, since

$$
\begin{aligned}
\left(\mathscr{Q}_{n}+\alpha\right)^{-1}\left(\mathscr{Q}_{n}+\alpha-\alpha\right) d & =d-\alpha\left(\mathscr{Q}_{n}+\alpha\right)^{-1} d \\
& =\left(\mathscr{Q}_{n}+\alpha-\alpha\right)\left(\mathscr{Q}_{n}+\alpha\right)^{-1} d .
\end{aligned}
$$

The second part of the result follows by the linearity of expectation $E$.

It will be shown that under certain assumptions the bias $\left\|E \mathscr{K} u_{n \alpha}-f\right\|_{\mu}$ can be bounded in terms of the continuous error $\left\|\mathscr{K} u_{\alpha}-f\right\|_{\mu}$, which was estimated in Section 3. We will use the fact that

$$
\left|\left\|E \mathscr{K} u_{n \alpha}-f\right\|_{\mu}-\left\|\mathscr{K} u_{\alpha}-f\right\|_{\mu}\right| \leq\left\|E \mathscr{K} u_{n \alpha}-\mathscr{K} u_{\alpha}\right\|_{\mu}
$$

and estimate the right-hand side.

By Lemma 4.1,

$$
\begin{aligned}
E \mathscr{K} u_{n \alpha}-\mathscr{K} u_{\alpha}= & \left(\mathscr{Q}_{n}+\alpha\right)^{-1} \mathscr{Q}_{n} f-(\mathscr{Q}+\alpha)^{-1} \mathscr{Q} f \\
= & \left(\mathscr{Q}_{n}+\alpha\right)^{-1} \mathscr{Q}_{n} f-(\mathscr{Q}+\alpha)^{-1} \mathscr{Q}_{n} f \\
& +(\mathscr{Q}+\alpha)^{-1} \mathscr{Q}_{n} f-(\mathscr{Q}+\alpha)^{-1} \mathscr{Q} f \\
= & (\mathscr{Q}+\alpha)^{-1}\left(\mathscr{Q}-\mathscr{Q}_{n}\right)\left(\mathscr{Q}_{n}+\alpha\right)^{-1} \mathscr{Q}_{n} f-(\mathscr{Q}+\alpha)^{-1}\left(\mathscr{Q}-\mathscr{Q}_{n}\right) f \\
= & (\mathscr{Q}+\alpha)^{-1}\left(\mathscr{Q}-\mathscr{Q}_{n}\right)\left(E \mathscr{K} u_{n \alpha}-f\right) .
\end{aligned}
$$


This working shows that $(\mathscr{Q}+\alpha)^{-1}\left(\mathscr{Q}-\mathscr{Q}_{n}\right): H \rightarrow H$, for if $g \in H$, let $f=$ $(1 / \alpha)\left(\mathscr{Q}_{n}+\alpha\right) g$. Then

$$
\begin{aligned}
(\mathscr{Q}+\alpha)^{-1}\left(\mathscr{Q}-\mathscr{Q}_{n}\right) g & =(\mathscr{Q}+\alpha)^{-1}\left(\mathscr{Q}-\mathscr{Q}_{n}\right)\left(\mathscr{Q}_{n}+\alpha\right)^{-1} \alpha f \\
& =(\mathscr{Q}+\alpha)^{-1}\left(\mathscr{Q}-\mathscr{Q}_{n}\right)\left(f-E \mathscr{K} u_{n \alpha}\right) \\
& =\mathscr{K} u_{\alpha}-E \mathscr{K} u_{n \alpha}
\end{aligned}
$$

and both $\mathscr{K} u_{\alpha}$ and $E \mathscr{K} u_{n \alpha}$ belong in $H$.

Now from above,

$$
\begin{aligned}
E \mathscr{K} u_{n \alpha}-f & =E \mathscr{K} u_{n \alpha}-\mathscr{K} u_{\alpha}+\mathscr{K} u_{\alpha}-f \\
& =(\mathscr{Q}+\alpha)^{-1}\left(\mathscr{Q}-\mathscr{Q}_{n}\right)\left(E \mathscr{K} u_{n \alpha}-f\right)+\mathscr{K} u_{\alpha}-f
\end{aligned}
$$

and hence,

$$
\left[I-(\mathscr{Q}+\alpha)^{-1}\left(\mathscr{Q}-\mathscr{Q}_{n}\right)\right]\left(E \mathscr{K} u_{n \alpha}-f\right)=\mathscr{K} u_{\alpha}-f
$$

Note that $I-(\mathscr{Q}+\alpha)^{-1}\left(\mathscr{Q}-\mathscr{Q}_{n}\right)$ has an inverse, since

$$
\begin{aligned}
I-(\mathscr{Q}+\alpha)^{-1}\left(\mathscr{Q}-\mathscr{Q}_{n}\right) & =(\mathscr{Q}+\alpha)^{-1}\left(\mathscr{Q}+\alpha-\mathscr{Q}+\mathscr{Q}_{n}\right) \\
& =(\mathscr{Q}+\alpha)^{-1}\left(\mathscr{Q}_{n}+\alpha\right)
\end{aligned}
$$

and this has inverse $\left(\mathscr{Q}_{n}+\alpha\right)^{-1}(\mathscr{Q}+\alpha)$. Hence, if the sum converges in $H_{\mu}$ for some $\mu$, then

$$
\begin{aligned}
E \mathscr{K} u_{n \alpha}-f & =\left[I-(\mathscr{Q}+\alpha)^{-1}\left(\mathscr{Q}-\mathscr{Q}_{n}\right)\right]^{-1}\left(\mathscr{K} u_{\alpha}-f\right) \\
& =\mathscr{K} u_{\alpha}-f+\sum_{l=1}^{\infty}\left[(\mathscr{Q}+\alpha)^{-1}\left(\mathscr{Q}-\mathscr{Q}_{n}\right)\right]^{l}\left(\mathscr{K} u_{\alpha}-f\right)
\end{aligned}
$$

and so

$$
E \mathscr{K} u_{n \alpha}-\mathscr{K} u_{\alpha}=\sum_{l=1}^{\infty}\left[(\mathscr{Q}+\alpha)^{-1}\left(\mathscr{Q}-\mathscr{Q}_{n}\right)\right]^{l}\left(\mathscr{K} u_{\alpha}-f\right) .
$$

We need to show that this is small in the $\mu$-norm. Intuitively, the difficulty lies in the fact that the parameters $\alpha$ and $n$ have opposing effects. Clearly, for $\mathscr{K} u_{\alpha}$ to converge to $f$, we require that $\alpha \rightarrow 0$ as $n \rightarrow \infty$. Now as $n \rightarrow \infty, \mathscr{Q}-\mathscr{Q}_{n}$ in some sense approaches 0 . However, as $\alpha \rightarrow 0,(\mathscr{Q}+\alpha)^{-1}$ becomes unbounded. Thus, in order that $(\mathscr{Q}+\alpha)^{-1}\left(\mathscr{Q}-\mathscr{Q}_{n}\right)$ approach 0 , we can expect that $\alpha$ must tend to 0 at some specific rate.

LEMMA 4.2. If $\mu<2-\nu-1 / 2 p$, then for all $g \in H_{\nu}$,

$$
\left\|(\mathscr{Q}+\alpha)^{-1}\left(\mathscr{Q}-\mathscr{Q}_{n}\right) g\right\|_{\mu} \leq \sqrt{c_{2}}(\nu+\mu) k_{n}\|g\|_{\nu} \alpha^{-(\nu+\mu+1 / 2 p) / 2} .
$$

Proof. Since $H$ is dense in $H_{\nu}$, it suffices to verify the inequality for all $g \in H$. By definition,

$$
\begin{aligned}
\left\|(\mathscr{Q}+\alpha)^{-1}\left(\mathscr{Q}-\mathscr{Q}_{n}\right) g\right\|_{\mu}^{2} & =\sum_{i=1}^{\infty} \frac{\left((\mathscr{Q}+\alpha)^{-1}\left(\mathscr{Q}-\mathscr{Q}_{n}\right) g, \phi_{i}\right)^{2}}{\lambda_{i}^{\mu}} \\
& =\sum_{i=1}^{\infty} \frac{\left(\left(\mathscr{Q}-\mathscr{Q}_{n}\right) g, \phi_{i}\right)^{2}}{\left(\lambda_{i}+\alpha\right)^{2} \lambda_{i}^{\mu}}
\end{aligned}
$$


Now,

$$
\left(\left(\mathscr{Q}-\mathscr{Q}_{n}\right) g, \phi_{i}\right)=\lambda_{i}\left(\int_{0}^{1} g \phi_{i}-\frac{1}{n} \sum_{j=1}^{n} g\left(x_{j}\right) \phi_{i}\left(x_{j}\right)\right),
$$

and hence by Assumption (2.3),

$$
\begin{aligned}
\left|\left(\left(\mathscr{Q}-\mathscr{Q}_{n}\right) g, \phi_{i}\right)\right| & \leq \lambda_{i} k_{n}\|g\|_{\nu}\left\|\phi_{i}\right\|_{\nu} \\
& =\lambda_{i} k_{n}\|g\|_{\nu}\left(\lambda_{i}\right)^{-\nu / 2}=\lambda_{i}^{1-\nu / 2} k_{n}\|g\|_{\nu} .
\end{aligned}
$$

Therefore,

$$
\left\|(\mathscr{Q}+\alpha)^{-1}\left(\mathscr{Q}-\mathscr{Q}_{n}\right) g\right\|_{\mu}^{2} \leq k_{n}^{2}\|g\|_{\nu}^{2} \sum_{i=1}^{\infty} \frac{\lambda_{i}^{2-\nu-\mu}}{\left(\lambda_{i}+\alpha\right)^{2}} .
$$

By Lemma (3.1), since $0<\nu+\mu<2-1 / 2 p$,

$$
\sum_{i=1}^{\infty} \frac{\lambda_{i}^{2-\nu-\mu}}{\left(\lambda_{i}+\alpha\right)^{2}} \leq c_{2}(\nu+\mu) \alpha^{-\nu-\mu-1 / 2 p}
$$

This proves the lemma.

LEMMA 4.3. Suppose $\nu<1-1 / 4 p$ and $\mu<2-\nu-1 / 2 p$. If $\alpha=\alpha(n) \rightarrow 0$ and $k_{n} \alpha^{-\nu-1 / 4 p} \rightarrow 0$ as $n \rightarrow \infty$, then for all $n$ sufficiently large, the resolvent

$$
R_{n \alpha}=\sum_{l=1}^{\infty}\left[(\mathscr{Q}+\alpha)^{-1}\left(\mathscr{Q}-\mathscr{Q}_{n}\right)\right]^{l}
$$

is a bounded operator $H_{\nu} \rightarrow H_{\mu}$, and for all $g \in H_{\nu}$,

$$
\left\|R_{n \alpha} g\right\|_{\mu} \leq \sqrt{c_{2}}(\nu+\mu) k_{n} \alpha^{-(\nu+\mu+1 / 2 p) / 2}\|g\|_{\nu} .
$$

Proof. If the sum converges, then for any $g \in H_{\nu}$,

$$
\left\|R_{n \alpha} g\right\|_{\mu} \leq \sum_{l=1}^{\infty}\left\|\left[(\mathscr{Q}+\alpha)^{-1}\left(\mathscr{Q}-\mathscr{Q}_{n}\right)\right]^{l} g\right\|_{\mu} .
$$

Since $\nu<1-1 / 4 p$, we can let $\mu=\nu$ in Lemma 4.2 to obtain

$$
\left\|(\mathscr{Q}+\alpha)^{-1}\left(\mathscr{Q}-\mathscr{Q}_{n}\right) g\right\|_{\nu} \leq \sqrt{c_{2}}(2 \nu) k_{n}\|g\|_{\nu} \alpha^{-\nu-1 / 4 p} .
$$

Hence, by induction,

$$
\begin{aligned}
\left\|\left[(\mathscr{Q}+\alpha)^{-1}\left(\mathscr{Q}-\mathscr{Q}_{n}\right)\right]^{l} g\right\|_{\mu} \\
\quad \leq \sqrt{c_{2}}(\mu+\nu) k_{n} \alpha^{-(\nu+\mu+1 / 2 p) / 2}\|g\|_{\nu}\left(\sqrt{c_{2}}(2 \nu) k_{n} \alpha^{-\nu-1 / 4 p}\right)^{l-1} .
\end{aligned}
$$

Since $k_{n} \alpha^{-\nu-1 / 4 p} \rightarrow 0$, we have

$$
\sqrt{c_{2}}(2 \nu) k_{n} \alpha^{-\nu-1 / 4 p}<1 / 2
$$

for all $n$ sufficiently large. Therefore, for such $n$,

$$
\begin{aligned}
\sum_{l=1}^{\infty} \| & {\left[(\mathscr{Q}+\alpha)^{-1}\left(\mathscr{Q}-\mathscr{Q}_{n}\right)\right]^{l} g \| } \\
& =\sqrt{c_{2}}(\nu+\mu) k_{n} \alpha^{-(\nu+\mu+1 / 2 p) / 2}\|g\|_{\nu}\left(1-\sqrt{c_{2}}(2 \nu) k_{n} \alpha^{-\nu-1 / 4 p}\right)^{-1} \\
& =\sqrt{c_{2}}(\nu+\mu) k_{n} \alpha^{-(\nu+\mu+1 / 2 p) / 2}\|g\|_{\nu}(1+o(1))
\end{aligned}
$$

Since by definition $\sqrt{c_{2}}(\nu+\mu)=\sqrt{c_{2}}(\nu+\mu)(1+o(1))$, the lemma follows. 
Applying Lemma 4.3 to (4.1) gives

$$
\begin{aligned}
& \|\| E \mathscr{K} u_{n \alpha}-f\left\|_{\mu}-\right\| \mathscr{K} u_{\alpha}-f \|_{\mu} \mid \\
& \quad \leq\left\|E \mathscr{K} u_{n \alpha}-\mathscr{K} u_{\alpha}\right\|_{\mu} \\
& \quad \leq \sqrt{c_{2}}(\nu+\mu) k_{n} \alpha^{-(\nu+\mu+1 / 2 p) / 2}\left\|\mathscr{K} u_{\alpha}-f\right\|_{\nu} .
\end{aligned}
$$

By Theorem 3.1, if $f \in H_{s}, s \geq \max \{\nu, \mu\}$, then the right-hand side $e(\alpha)$ of (4.2) satisfies

$$
e(\alpha) \lesssim k_{n} \alpha^{-\nu-1 / 4 p} \alpha^{\nu / 2-\mu / 2} \begin{cases}o(1), & s=\nu \\ \alpha^{s / 2-\nu / 2}\|f\|_{s}, & \nu<s \leq \nu+2, \\ \alpha\|f\|_{\nu+2}, & s \geq \nu+2\end{cases}
$$

By examining the exponents, if $\mu \leq \nu$ then

$$
e(\alpha) \lesssim k_{n} \alpha^{-\nu-1 / 4 p} \begin{cases}\alpha^{s / 2-\mu / 2}, & \nu \leq s \leq \mu+2 \\ \alpha, & s \geq \mu+2\end{cases}
$$

and if $\mu>\nu$ then

$$
e(\alpha) \lesssim \begin{cases}k_{n} \alpha^{-\nu-1 / 4 p} \alpha^{s / 2-\mu / 2}, & \mu \leq s \leq \nu+2 \\ k_{n} \alpha^{-\nu / 2-\mu / 2-1 / 4 p} \alpha^{s / 2-\mu / 2}, & \nu+2 \leq s \leq \mu+2 \\ k_{n} \alpha^{-\nu / 2-\mu / 2-1 / 4 p} \alpha, & s \geq \mu+2\end{cases}
$$

Hence, from (4.2), if as $n \rightarrow \infty$

$$
k_{n} \alpha^{-\nu-1 / 4 p} \rightarrow 0 \text { when } \mu \leq \nu \text { or when } \mu>\nu, \mu \leq s \leq \nu+2
$$

and

$$
k_{n} \alpha^{-\nu / 2-\mu / 2-1 / 4 p} \rightarrow 0 \quad \text { when } \mu>\nu, s>\nu+2
$$

then

$$
\left|\left\|E \mathscr{K} u_{n \alpha}-f\right\|_{\mu}-\left\|\mathscr{K} u_{\alpha}-f\right\|_{\mu}\right|= \begin{cases}o\left(\alpha^{s / 2-\mu / 2}\right), & s \leq \mu+2 \\ o(\alpha), & s \geq \mu+2\end{cases}
$$

By squaring (4.5) and using Theorem 3.1, we have the following estimate of the bias squared.

THEOREM 4.1. Suppose that Assumptions 2.1, 2.2 and 2.3 hold, and let $f \in H_{s}$ where $s \geq \max \{\nu, \mu\}$ and $\mu<2-\nu-1 / 2 p$. If $\alpha=\alpha(n) \rightarrow 0$ as $n \rightarrow \infty$ so that (4.3) and (4.4) hold, then

$$
\left|\left\|E u_{n \alpha}-\mathscr{K}^{\dagger} f\right\|_{\mu}^{2}-\left\|u_{\alpha}-\mathscr{K}^{\dagger} f\right\|_{\mu}^{2}\right|= \begin{cases}o\left(\alpha^{s-\mu}\right), & s \leq \mu+2 \\ o\left(\alpha^{2}\right), & s \geq \mu+2\end{cases}
$$

From above, if in fact $\alpha$ satisfies

$$
k_{n} \alpha^{-\nu-1 / 4 p} \approx \alpha^{d / 2}, \quad d>0
$$

then the error estimate $o\left(\alpha^{s-\mu}\right)$ in the case $s \leq \mu+2, \mu \leq \nu$ can be strengthened to $O\left(\alpha^{s-\mu+d}\right)$. Then, for the function $f$ defined by (3.4),

$$
\left\|E u_{n \alpha}-\mathscr{K}^{\dagger} f\right\|_{\mu}^{2} \leq O\left(\alpha^{\bar{s}-\mu+e}\right),
$$

where $e=\min \{d, 1+\mu / 2-\bar{s} / 2, \bar{s}-\mu\}$. This shows that for the case $\bar{s} \leq \mu+2$ it is in general impossible to bound the bias as follows: For any $\varepsilon>0$,

$$
\left\|E u_{n \alpha}-\mathscr{K}^{\dagger} f\right\|_{\mu}^{2} \gtrsim \alpha^{\bar{s}-\mu+\varepsilon} .
$$

Nevertheless we can prove the following. 
THEOREM 4.2. Suppose that Assumptions 2.1, 2.2 and 2.3 hold, and let $f \in$ $H_{s}$, where $s \geq \max \{\nu, \mu\}, \bar{s} \leq \mu+2$ and $\mu<2-\nu-1 / 2 p$. For any $\varepsilon>0$ there exists a sequence $\alpha=\alpha(n) \rightarrow 0$ as $n \rightarrow \infty$ such that

$$
\left\|E u_{n \alpha}-\mathscr{K}^{\dagger} f\right\|_{\mu}^{2} \gtrsim \alpha^{\bar{s}-\mu+\varepsilon} .
$$

Proof. By Theorems 3.1 and 3.2, for any $\varepsilon>0$ there exists an infinite sequence of $\alpha \rightarrow 0$ such that

$$
\frac{\left\|\mathscr{K} u_{\alpha}-f\right\|_{\nu}^{2}}{\left\|\mathscr{K} u_{\alpha}-f\right\|_{\mu}^{2}} \lesssim \begin{cases}\alpha^{\mu-\nu-2 \varepsilon}, & \bar{s} \leq \min \{\nu, \mu\}+2 \\ \alpha^{\mu+2-\bar{s}-\varepsilon}, & \min \{\nu, \mu\}+2<\bar{s} \leq \mu+2\end{cases}
$$

Substituting these inequalities into (4.2) yields

$$
\left|\left\|E \mathscr{K} u_{n \alpha}-f\right\|_{\mu}-\left\|\mathscr{K} u_{\alpha}-f\right\|_{\mu}\right| \leq a\left\|\mathscr{K} u_{\alpha}-f\right\|_{\mu}
$$

where

$$
a \lesssim \begin{cases}k_{n} \alpha^{-\nu-1 / 4 p-\varepsilon}, & \bar{s} \leq \min \{\nu, \mu\}+2, \\ k_{n} \alpha^{1-\nu / 2-\bar{s} / 2-1 / 4 p-\varepsilon / 2}, & \min \{\nu, \mu\}+2<\bar{s} \leq \mu+2 .\end{cases}
$$

Now the sequence of $\alpha \rightarrow 0$ can be chosen to make $a=o(1)$. For, if the sequence converges to 0 too quickly, then by simply repeating an appropriate number of terms, a new sequence can be constructed which does satisfy the constraints. The result follows from (4.7) and Theorem 3.2.

THEOREM 4.3. Suppose that Assumptions 2.1, 2.2 and 2.3 hold, and let $f \in$ $H_{s}$, where $s>\max \{\nu, \mu\}$ and $\mu<2-\nu-1 / 2 p$. If $f$ satisfies condition (2.2) and $\alpha=\alpha(n) \rightarrow 0$ as $n \rightarrow \infty$ so that (2.3) and (2.4) hold, then

$$
\left\|E u_{n \alpha}-\mathscr{K}^{\dagger} f\right\|_{\mu}=\left\|u_{\alpha}-\mathscr{K}^{\dagger} f\right\|_{\mu}(1+o(1)) \text {. }
$$

Proof. By Theorems 3.1 and 3.3, the bounds (4.6) and hence (4.7) are valid for all sufficiently small $\alpha$. This proves the result if $\bar{s} \leq \mu+2$. If $\bar{s}>\mu+2$, the result follows from Theorems 4.1 and 3.1 .

5. Estimate of the Variance. Although not essential, the first result is of interest here.

LEMMA 5.1. Let

$$
f_{x}(y)=\frac{1}{n}\left(\mathscr{Q}_{n}+\alpha\right)^{-1} Q(x, y)
$$

and

$$
g_{x}(y)=\frac{1}{n}\left(\mathscr{Q}_{n}+\alpha\right)^{-1} Q(y, x)
$$

where $\left(\mathscr{Q}_{n}+\alpha\right)^{-1}$ acts on $Q$ as a function of its first variable. Then for each $k=1,2, \ldots, n$,

$$
f_{x_{k}}(y)=g_{x_{k}}(y)=\mathscr{K} u_{n \alpha}^{k}(y) .
$$

Here, $u_{n \alpha}^{k}$ is the regularized solution with data

$$
\mathbf{d}^{k}=(0, \ldots, 0,1,0, \ldots, 0),
$$

where the 1 is in the $k$ th place. 
Proof. By Lemma 4.1,

$$
\begin{aligned}
\mathscr{K} u_{n \alpha}^{k}(y) & =\left(\mathscr{Q}_{n}+\alpha\right)^{-1} \mathscr{Q}_{n} d^{k} \\
& =\left(\mathscr{Q}_{n}+\alpha\right)^{-1} \frac{1}{n} \sum_{i=1}^{n} Q\left(y, x_{i}\right) \mathbf{d}_{i}^{k} \\
& =\frac{1}{n}\left(\mathscr{Q}_{n}+\alpha\right)^{-1} Q\left(y, x_{k}\right)=g_{x_{k}}(y) .
\end{aligned}
$$

Also, from Lemma 4.1,

$$
\begin{aligned}
\mathscr{K} u_{n \alpha}^{k}(y) & =\sum_{i=1}^{n} Q\left(x_{i}, y\right)\left(Q_{n}+\alpha n I\right)^{-1} \mathbf{d}_{i}^{k} \\
& =\sum_{i=1}^{n}\left(Q_{n}+\alpha n I\right)_{k i}^{-1} Q\left(x_{i}, y\right) \\
& =\frac{1}{n}\left(\mathscr{Q}_{n}+\alpha\right)^{-1} Q\left(x_{k}, y\right)=f_{x_{k}}(y)
\end{aligned}
$$

Now we derive the following expression for the variance (1.7).

LEMMA 5.2. We have

$$
E\left\|\mathscr{K} u_{n \alpha}-E \mathscr{K} u_{n \alpha}\right\|_{\mu}^{2}=\sigma^{2} \sum_{k=1}^{n}\left\|F_{k}\right\|_{\mu}^{2},
$$

where $F_{k}=f_{x_{k}}=g_{x_{k}}=\mathscr{K} u_{n \alpha}^{k}$, from Lemma 5.1.

Proof. Using Lemma 4.1 and Assumption 2.1 about the errors $\varepsilon_{i}=d_{i}-f\left(x_{i}\right)$, we get

$$
\begin{aligned}
E \| \mathscr{K} & u_{n \alpha}-E \mathscr{K} u_{n \alpha} \|_{\mu}^{2} \\
& =E\left\|\left(\mathscr{Q}_{n}+\alpha\right)^{-1} \mathscr{Q}_{n}(d-f)\right\|_{\mu}^{2} \\
& =E\left\|\left(\mathscr{Q}_{n}+\alpha\right)^{-1} \frac{1}{n} \sum_{i=1}^{n} Q\left(\cdot, x_{i}\right) \varepsilon_{i}\right\|_{\mu}^{2} \\
& =\sum_{i, k=1}^{n}\left(\frac{1}{n}\left(\mathscr{Q}_{n}+\alpha\right)^{-1} Q\left(\cdot, x_{i}\right), \frac{1}{n}\left(\mathscr{Q}_{n}+\alpha\right)^{-1} Q\left(\cdot, x_{j}\right)\right)_{\mu} E \varepsilon_{i} \varepsilon_{k} \\
& =\sigma^{2} \sum_{k=1}^{n}\left\|F_{k}\right\|_{\mu}^{2} .
\end{aligned}
$$

Of the three possible forms of $F_{k}$, we will use

$$
F_{k}(y)=g_{x_{k}}(y)=\frac{1}{n}\left(\mathscr{Q}_{n}+\alpha\right)^{-1} Q\left(y, x_{k}\right)
$$

It will be shown that $\left(\mathscr{Q}_{n}+\alpha\right)^{-1} Q\left(y, x_{k}\right)$ can be approximated by $(\mathscr{Q}+\alpha)^{-1} Q\left(y, x_{k}\right)$, and this will lead to an estimate of the variance.

Corresponding to Lemma 5.1, it is not hard to show that for all $x, y \in[0,1]$,

$$
(\mathscr{Q}+\alpha)^{-1} Q(x, y)=(\mathscr{Q}+\alpha)^{-1} Q(y, x),
$$

where $(\mathscr{Q}+\alpha)^{-1}$ acts on $Q$ as a function of its first variable. Note also that $(\mathscr{Q}+\alpha)^{-1} Q\left(y, x_{k}\right)$ can be thought of as $\mathscr{K} u_{\alpha}(y)=(\mathscr{Q}+\alpha)^{-1} \mathscr{Q} f(y)$ with data 
$f(x)=\delta\left(x-x_{k}\right)$, a Dirac delta "function". In the case of data smoothing with $W=$ $W^{m, 2}[0,1],(\mathscr{Q}+\alpha)^{-1} Q(x, y)$ is the Green's function of a certain linear differential operator. This fact was exploited by Cox [4].

Proceeding as in Section 4,

$$
\begin{aligned}
& \left(\mathscr{Q}_{n}+\alpha\right)^{-1} Q\left(y, x_{k}\right)-(\mathscr{Q}+\alpha)^{-1} Q\left(y, x_{k}\right) \\
& \quad=(\mathscr{Q}+\alpha)^{-1}\left(\mathscr{Q}-\mathscr{Q}_{n}\right)\left(\mathscr{Q}_{n}+\alpha\right)^{-1} Q\left(y, x_{k}\right)
\end{aligned}
$$

and hence

$$
\left[I-(\mathscr{Q}+\alpha)^{-1}\left(\mathscr{Q}-\mathscr{Q}_{n}\right)\right]\left(\mathscr{Q}_{n}+\alpha\right)^{-1} Q\left(y, x_{k}\right)=(\mathscr{Q}+\alpha)^{-1} Q\left(y, x_{k}\right) .
$$

Since

$$
\begin{aligned}
(\mathscr{Q}+\alpha)^{-1} Q\left(y, x_{k}\right) & =\frac{1}{\alpha}(\mathscr{Q}+\alpha-\mathscr{Q})(\mathscr{Q}+\alpha)^{-1} Q\left(y, x_{k}\right) \\
& =\frac{1}{\alpha} Q\left(y, x_{k}\right)-\frac{1}{\alpha} \mathscr{Q}(\mathscr{Q}+\alpha)^{-1} Q\left(y, x_{k}\right),
\end{aligned}
$$

$(\mathscr{Q}+\alpha)^{-1} Q\left(y, x_{k}\right)$ belongs to $H_{1}$ and hence to $H_{\nu}$. Then by Lemma 4.3,

$$
\left(\mathscr{Q}_{n}+\alpha\right)^{-1} Q\left(y, x_{k}\right)-(\mathscr{Q}+\alpha)^{-1} Q\left(y, x_{k}\right)=R_{n \alpha}(\mathscr{Q}+\alpha)^{-1} Q\left(y, x_{k}\right)
$$

and

$$
\begin{aligned}
& \left\|\left(\mathscr{Q}_{n}+\alpha\right)^{-1} Q\left(y, x_{k}\right)-(\mathscr{Q}+\alpha)^{-1} Q\left(y, x_{k}\right)\right\|_{\mu} \\
& \quad \leq \sqrt{c_{2}}(\nu+\mu) k_{n} \alpha^{-(\nu+\mu+1 / 2 p) / 2}\left\|(\mathscr{Q}+\alpha)^{-1} Q\left(y, x_{k}\right)\right\|_{\nu} .
\end{aligned}
$$

From the expansion of $Q(x, y)$ it is easy to verify that

$$
(\mathscr{Q}+\alpha)^{-1} Q\left(y, x_{k}\right)=\sum_{i=1}^{\infty}\left(\frac{\lambda_{i}}{\lambda_{i}+\alpha}\right) \phi_{i}(y) \phi_{i}\left(x_{k}\right)
$$

where the sum converges uniformly for any $\alpha>0$. Therefore,

$$
\left\|(\mathscr{Q}+\alpha)^{-1} Q\left(y, x_{k}\right)\right\|_{\mu}^{2}=\sum_{i=1}^{\infty}\left(\frac{\lambda_{i}}{\lambda_{i}+\alpha}\right)^{2} \frac{\phi_{i}^{2}\left(x_{k}\right)}{\lambda_{i}^{\mu}} .
$$

By Assumption 2.3,

$$
\begin{aligned}
\left|\frac{1}{n} \sum_{k=1}^{n} \phi_{i}^{2}\left(x_{k}\right)-\int_{0}^{1} \phi_{i}^{2}(x) d x\right| & =\left|\frac{1}{n} \sum_{k=1}^{n} \phi_{i}^{2}\left(x_{k}\right)-1\right| \\
& \leq k_{n}\left\|\phi_{i}\right\|_{\nu}^{2}=k_{n} \lambda_{i}^{-\nu} .
\end{aligned}
$$

Hence, combining (5.2) and (5.3), we get

$$
\begin{aligned}
& \left|\frac{1}{n} \sum_{k=1}^{n}\left\|(\mathscr{Q}+\alpha)^{-1} Q\left(y, x_{k}\right)\right\|_{\mu}^{2}-\sum_{i=1}^{\infty}\left(\frac{\lambda_{i}}{\lambda_{i}+\alpha}\right)^{2} \frac{1}{\lambda_{i}^{\mu}}\right| \\
& \leq k_{n} \sum_{i=1}^{\infty}\left(\frac{\lambda_{i}}{\lambda_{i}+\alpha}\right)^{2} \lambda_{i}^{-\nu-\mu} .
\end{aligned}
$$

Let

$$
A_{k}=\left\|\left(\mathscr{Q}_{n}+\alpha\right)^{-1} Q\left(y, x_{k}\right)\right\|_{\mu}, \quad \cdot \quad B_{k}=\left\|(\mathscr{Q}+\alpha)^{-1} Q\left(y, x_{k}\right)\right\|_{\mu}
$$

and

$$
C_{k}=\sqrt{c_{2}}(\nu+\mu) k_{n} \alpha^{-(\nu+\mu+1 / 2 p) / 2}\left\|(\mathscr{Q}+\alpha)^{-1} Q\left(y, x_{k}\right)\right\|_{\nu}
$$


Using (5.1), we have

$$
\begin{aligned}
\left|\sum_{k=1}^{n} A_{k}^{2}-\sum_{k=1}^{n} B_{k}^{2}\right| & \leq \sum_{k=1}^{n}\left|A_{k}-B_{k}\right|^{2}+2 \sum_{k=1}^{n} B_{k}\left|A_{k}-B_{k}\right| \\
& \leq \sum_{k=1}^{n} C_{k}^{2}+2 \sum_{k=1}^{n} B_{k} C_{k} \\
& \leq \sum_{k=1}^{n} C_{k}^{2}+2\left(\sum_{k=1}^{n} B_{k}^{2}\right)^{1 / 2}\left(\sum_{k=1}^{n} C_{k}^{2}\right)^{1 / 2}
\end{aligned}
$$

Now from (5.4) and Lemma 3.1, since $\mu<2-1 / 2 p$ and $\nu+\mu<2-1 / 2 p$, we have

$$
\begin{aligned}
\frac{1}{n} \sum_{k=1}^{n} B_{k}^{2} & \geq c_{1}(\mu) \alpha^{-\mu-1 / 2 p}-k_{n} c_{2}(\nu+\mu) \alpha^{-\nu-\mu-1 / 2 p} \\
& =c_{1}(\mu) \alpha^{-\mu-1 / 2 p}(1-\varepsilon)
\end{aligned}
$$

where

$$
\varepsilon=\left(c_{2}(\nu+\mu) / c_{1}(\mu)\right) k_{n} \alpha^{-\nu}=o(1),
$$

since $k_{n} \alpha^{-\nu-1 / 4 p} \rightarrow 0$. Also, from (5.4) with $\mu=\nu$, and from Lemma 3.1, since $\nu<1-1 / 4 p$, we have

$$
\begin{aligned}
\frac{1}{n} \sum_{k=1}^{n} C_{k}^{2} & =c_{2}(\nu+\mu) k_{n}^{2} \alpha^{-(\nu+\mu+1 / 2 p)} \frac{1}{n} \sum_{k=1}^{n}\left\|(\mathscr{Q}+\alpha)^{-1} Q\left(y, x_{k}\right)\right\|_{\nu}^{2} \\
& \leq c_{2}(\nu) c_{2}(\nu+\mu) k_{n}^{2} \alpha^{-2 \nu-\mu-1 / p}(1+\delta)
\end{aligned}
$$

where

$$
\delta=\left(c_{2}(2 \nu) / c_{2}(\nu)\right) k_{n} \alpha^{-\nu}=o(1)
$$

since $k_{n} \alpha^{-\nu-1 / 4 p} \rightarrow 0$.

Substituting (5.6) and (5.7), we find that

$$
\begin{aligned}
\sum_{k=1}^{n} C_{k}^{2} / \sum_{k=1}^{n} B_{k}^{2} & \leq\left(c_{2}(\nu) c_{2}(\nu+\mu) / c_{1}(\mu)\right) k_{n}^{2} \alpha^{-2 \nu-1 / 2 p}(1+o(1)) \\
& =o(1)
\end{aligned}
$$

since $k_{n} \alpha^{-\nu-1 / 4 p} \rightarrow 0$. Using this in (5.5) yields

$$
\sum_{k=1}^{n} A_{k}^{2}=\sum_{k=1}^{n} B_{k}^{2}(1+o(1))
$$

From (5.4) and Lemma 3.1, we have

$$
c_{1}(\mu) \alpha^{-\mu-1 / 2 p} \leq \frac{1}{n} \sum_{k=1}^{n} B_{k}^{2} \leq c_{2}(\mu) \alpha^{-\mu-1 / 2 p}
$$

since by definition, $c_{1}(\mu)=c_{1}(\mu)(1+o(1))$ and $c_{2}(\mu)=c_{2}(\mu)(1+o(1))$.

Combining (5.8), (5.9) and Lemma 5.2, we have the following estimate of the variance. 
THEOREM 5.1. Suppose Assumptions 2.1, 2.2 and 2.3 hold and $\mu<2-\nu-1 / 2 p$. If $\alpha=\alpha(n) \rightarrow 0$ and $k_{n} \alpha^{-\nu-1 / 4 p} \rightarrow 0$ as $n \rightarrow \infty$, then

$$
c_{1}(\mu) \frac{\sigma^{2}}{n} \alpha^{-\mu-1 / 2 p} \leq E\left\|u_{n \alpha}-E u_{n \alpha}\right\|_{\mu}^{2} \leq c_{2}(\mu) \frac{\sigma^{2}}{n} \alpha^{-\mu-1 / 2 p} .
$$

School of Mathematical and Physical Sciences

Murdoch University

Murdoch, Western Australia, 6150

1. N. ARONSZAJn, "Theory of reproducing kernels," Trans. Amer. Math. Soc., v. 68, 1950, pp. 337-404.

2. J. Aubin, Applied Functional Analysis, Wiley, New York, 1979.

3. C. Bennett \& J. E. Gilbert, "Homogeneous algebras on the circle: II. Multipliers, Ditkin conditions," Ann. Inst. Fourier (Grenoble), v. 22, 1972, pp. 21-50.

4. D. D. Cox, "Asymptotics for $M$-type smoothing splines," Ann. Statist., v. 11, 1983, pp. $530-551$.

5. D. D. Cox, "Multivariate smoothing spline functions," SIAM J. Numer. Anal., v. 21, 1984, pp. 789-813.

6. D. D. Cox, Approximation of Method of Regularization Estimators, Technical Report No. 723, Dept. of Statistics, Univ. of Wisconsin-Madison, 1983.

7. P. CRAVEN \& G. WAHBA, "Smoothing noisy data with spline functions: Estimating the correct degree of smoothing by the method of generalized cross-validation," Numer. Math., v. 31, 1979, pp. 377-403.

8. J. W. HILGERS, "On the equivalence of regularization and certain reproducing kernel Hilbert space approaches for solving first kind problems," SIAM J. Numer. Anal., v. 13, 1976, pp. 172-184.

9. E. HILlE, "Introduction to general theory of reproducing kernels," Rocky Mountain J. Math., v. 2, 1972, pp. 321-368.

10. M. Z. NASHED \& G. WAHBA, "Generalized inverses in reproducing kernel spaces: An approach to regularization of linear operator equations," SIAM J. Math. Anal., v. 5, 1974, pp. 974-987.

11. F. NATTERER, "Error bounds for Tikhonov regularization in Hilbert scales," Applicable Anal., v. 18, 1984, pp. 29-37.

12. J. RICE \& M. ROSENBLATT, "Integrated mean squared error of a smoothing spline," $J$. Approx. Theory, v. 33, 1981, pp. 353-369.

13. J. RiCE \& M. RosenblatT, "Smoothing splines: regression, derivatives and deconvolution," Ann. Statist., v. 11, 1983, pp. 141-156.

14. F. RiEsz \& B. Sz.-NAGY, Functional Analysis, Ungar, New York, 1955.

15. P. SPECKMAN, "The asymptotic integrated mean square error for smoothing noisy data by splines," Numer. Math. (To appear.)

16. U. TipPenhauer, "Methoden zur Bestimmung von Reprokernen," J. Approx. Theory, v. 21, 1977, pp. 394-410.

17. H. TRIEBEL, Interpolation Theory, Function Spaces, Differential Operators, North-Holland, New York, 1978.

18. F. UTRERAS Diaz, "Sur le choix du paramètre d'adjustement dans le lissage par fonctions spline," Numer. Math., v. 34, 1980, pp. 15-28.

19. F. UTRERAS, "Natural spline functions, their associated eigenvalue problem," Numer. Math., v. 42, 1983, pp. $107-117$.

20. G. WAHBA, "Convergence rates of certain approximate solutions to Fredholm integral equations of the first kind," J. Approx. Theory, v. 7, 1973, pp. 167-185.

21. G. WAHBA, "Practical approximate solutions to linear operator equations when the data are noisy," SIAM J. Numer. Anal., v. 14, 1977, pp. 651-667.

22. G. WAHBA, "Constrained regularization for ill posed linear operator equations, with applications in meteorology and medicine," in Statistical Decision Theory and Related Topics: III, Vol. 2 (S. S. Gupta and J. O. Berger, eds.), Academic Press, New York, 1982. 